\title{
The chronological and social implications of the pottery from Jebel Moya (south-central
} Sudan)

Note: High resolution images are available on request. All images and captions are at the end of the document.

\begin{abstract}
Continued research at Jebel Moya shows that this burial and habitation site has a very long chronology and was the locus for a number of activities. This study presents the first comprehensive analysis of pottery from stratified contexts from the new field seasons, utilizing a statistical attribute approach that provides both clarity and avenues for further research. The stratigraphic sequence and radiometric dates show that the site was inhabited from at least the late $6^{\text {th }}$ millennium to 2000 years ago. Our analyses reveal previously unknown types of pottery and a wider range within assemblages. Overall, there is a longer period of mid-late Holocene habitation than previously recognised. Results are considered within a broader contextual and comparative approach with central Sudan, showing the importance of rethinking networks between south-central and central Sudan.

Highlights

1. Comprehensive re-examination of chronology shows the site has a long and complex history

2. Illustrated descriptions of pottery assemblage, particularly tools and motor actions

3. Identification of new vessels, techniques
\end{abstract}

Keywords: Sudan, pottery, agro-pastoralism, attribute analysis, Gezira Plain

\section{Introduction}

In this paper, we analyse the pottery from the 2017 and 2019 excavation seasons at the site of Jebel Moya (south-central Sudan) using single and co-occurring attribute analysis. Widely applied elsewhere in Africa (Haour et al., 2010; Keech McIntosh, 1995), the application of this technique remains in its infancy in the Sudan. It builds upon Brass' (2016) previous analyses of extant pottery sherds from Wellcome's excavations curated at the British Museum (BM), which had resulted in the first viable chronological reconstruction for the site. The excavated pottery is the first stratigraphically and statistically coherent reconstruction of the pottery sequencing for this part of the eastern Sahel (southern Gezira). It both extends the known intra- and interassemblage variability and has implications for the overall chronology. This assemblage is considered in conjunction with radiometric dates and stratigraphy, presenting the first integrated study of this complex site. The results (a) show that Jebel Moya was not merely a mortuary site, (b) the site was occupied from at least the $6^{\text {th }}$ millennium BC, (c) offer a secure dating sequence and (d) offer an insight into the community of practice as seen via ceramics.

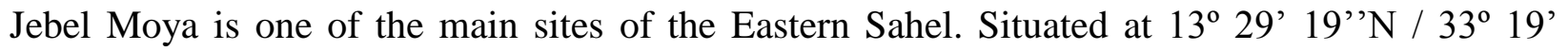
05' 'E in the southern Gezira Plain of south-central Sudan, two-fifths of the 10.4-hectare valley was originally excavated by Henry Wellcome from 1911-14 (Figure 1). Wellcome's expedition uncovered substantial mortuary activities, but there was no systematic study and storage of materials (Vella Gregory, 2020). It was only after the Second World War that Frank Addison (1949) published a report on the excavations, by which time material had been dispersed and field notes had been lost (for a historical discussion of excavation history see Brass 2016). 
Systematic fieldwork finally started in 2017 with The University College London - University of Khartoum - NCAM Expedition to the South Gezira (Sudan) (Brass et al., 2020, 2018c, 2018a, 2018b). Cumulatively, the site has yielded 3140 human burials (of which five have been stratigraphically excavated by the current mission) and the second oldest known occurrence of domesticated sorghum. Current excavations have identified areas of non-mortuary activity, making this one of the largest mortuary and habitation sites known in sub-Saharan Africa.

An AMS dating program has started encompassing the faunal, botanical and human skeletal remains from the two field seasons, with the radiometric results from the first season now known (Table 1a). It is backed by revisions made to the OSL dates on pottery samples from the British Museum collection, made possible by the taking of fresh soil samples (Table 1b). Three macrolevel phases of occupation are discernible from these radiometric dates, and these can be correlated with the macro-geological strata termed A-D in descending order:

1. Phase 1. This late $6^{\text {th }}$ millennium BC phase encompasses Stratum D and pottery Assemblage 1.

2. Phase 2. Two sub-phases are known, encompassing the $3^{\text {rd }}$ millennium $\mathrm{BC}$ and the mid$2^{\text {nd }}$ millennium BC. It is represented by pottery Assemblage 2 and is present in Stratum B. Burial activity occurred in this phase.

3. Phase 3. This $1^{\text {st }}$ millennium $B C$ to early $1^{\text {st }}$ millennium $A D$ phase encompasses strata $A$ and $\mathrm{B}$, and pottery Assemblage 3. Heavy burial activity occurred in this phase.

The presence of domesticated sorghum, processing querns and hammerstones means that for at least the last two recognised phases the occupants were agro-pastoralists (Brass et al., 2019). The examination of the pottery from the 2017 and 2019 seasons presented here extends the range of Phase 2 back to the $4^{\text {th }}$ and perhaps even the late $5^{\text {th }}$ millennium BC, through similarities in pottery between the inhabitants of the southern Gezira and the Central Sudan over $240 \mathrm{~km}$ to the north. It also proposes that Phase 3 began in the late $2^{\text {nd }}$ millennium $\mathrm{BC}$.

\section{Materials and methods}

The present study is a result of a thorough examination of known pottery from Wellcome's excavations and stratigraphically excavated assemblages. It explicitly employs attribute analysis, a method rooted in statistical analyses with the aim of understanding both the technological and social processes related to pottery production. Furthermore, it considers the legacy of historical approaches which continue to shape studies of Sudanese pottery. These approaches have assumed the status of accepted paradigms but, as this analysis shows, they are problematic.

The foundations for a Sudanese typology were laid down by A.J. Arkell's (1949, 1953) pioneering work at Khartoum, Shaheinab and el-Qoz. This consists of an undefined classificatory system based on visual impressions of decorative motifs (for a critique see Caneva, 1987: 233; Mohammed-Ali and Khabir, 2003: 29). Subsequent scholars have proposed alternative taxonomic classificatory schemes (Adams and Adams, 1991; Camps-Fabrer, 1966; Caneva, 1988, 1987; Gatto, 2006; Hays, 1971; Marks et al., 1985; Mohammed-Ali 1991; Mohammed-Ali and Khabir, 2003; Nordstrom, 1972; Winchell, 2013). Together, these focus on different 
elements with a patchwork of terminology that in many cases hinders the ability to recognise variation beyond restrictive and artificial categories.

Camps-Fabrer (1966) emphasised decorative pottery technologies such as rouletting rather than a sole focus on finished motif and surface treatments, although she did not propose methods for analysing the décor. T.R. Hays (1971) was the first to use the explicit Type Variety system in North-East Africa, which sorts pottery into hierarchically nested types and varieties based on the decorative style used. The Type Variety systems has not been widely adopted in Africa in general and Sudan in particular (for an exception, see Winchell, 2013). Subsequently, Nordstrom (1972) produced the first technological indices encompassing all then-known variables of fabric, techniques and decor which, however, failed to provide a chronological means of ordering due to the homogenous nature of his material from northern Nubia (Mohammed-Ali and Khabir, 2003: 51). Adams and Adams (1991) have highlighted the issues with such diverse typological categorisations, with no agreed means of standardisation. They worked out that archaeologists may have up a $10 \%$ divergence in their identification and categorisation of motifs even when using the same system. Despite these issues, it was these attempts at pottery typologies which provided the chronological backbone of Sudanese Holocene archaeological studies, which helps explain their continued influence despite the increased application and resolution of radiometric dating in the past three decades (Adams and Adams, 1991; Caneva, 1988; Gatto, 2006; Salvatori, 2012; Salvatori and Usai, 2008, 2007).

In an effort to provide a measure of standardisation, Isabella Caneva (1987) proposed a new system whereby the preferential order of variables is predetermined through standardisation. She later applied this to her Sudanese work (Caneva, 1988; Caneva and Marks, 1990). Its aim was to enable different scholars to repeat investigations without significant variations and to assist in establishing local and regional chronological sequences through the reconstruction of the internal evolution of decorative patterns. It gave precedence to "decorative techniques" as the primary method of analysis. Caneva downplayed the usefulness of tools in assessing spatial-temporal and cultural significance. In descending order of importance and with increasing levels of specificity in a tree-like typology, her typology emphasised technique $->$ implement $->$ element $->$ motif $->$ structure (Caneva 1987; Caneva and Marks 1990: 14).

Caneva's system partly moves away from the previous typological systems employed in the Sudan towards an attribute:vessel system but it remains structured within a typological framework. It has since become the dominant classificatory system in use in Saharan and Sudanese pottery studies (Caneva and Marks, 1990; Gatto, 2011a, 2011b; Jesse, 2010, 2002; Salvatori, 2012). This seemingly methodical approach relies heavily on motifs rather than the tools which made them. This results in a subjective focus on style (Haour et al., 2010), while overlooking the agency of potters and the wider social technological implications.

The present study acknowledges elements of Caneva's framework but eschews the tree-like typology. Aside from a considered statistical approach, we also employed a more rigorous classification of pottery, moving beyond problematic descriptions such as "red burnished ware", "wavy lines" or "dotted wavy line", what Haour et al. (2010: 3) termed "fossils directeurs of ambiguous definition" focusing as they do on convergent patterns. Following Keech McIntosh (1995), Mayor et al. (2005), MacDonald (2011) and others (Brass, 2016; Vella Gregory, 2017), 
the tool types and motor actions are identified to help: (a) minimise bias in determining the highest level grouping of attributes, as the variety and types of tools which are used to produce these motifs may reflect stylistic and technological diversity and/or social choice, and (b) discern temporally significant clusters through single and co-occurring attribute analyses. It is also acknowledged that these classificatory tools are a means towards understanding the ancient inhabitants of Jebel Moya, as opposed to previous approaches which treated the assemblage as an end in itself.

Jebel Moya's ascribed marginality created the artificial problem of comparanda. This was compounded by a broader typological approach to pottery that focused on superficial categories. Thus, sherds from Levels 2 and 3 in Rabak, a site c. $70 \mathrm{~km}$ away on the banks of the White Nile, were seen as 'strikingly similar' to material from Jebel Moya (el Mahi and Haaland, 1984; Haaland, 1987, 1984). Furthermore, a shell from Level 2 was dated to 3378 - 2909 BC Table 2). In turn, this was used to provide a date for Jebel Moya (Gerharz, 1994). The shell was not found in association with any material and no correction was made to account for the freshwater reservoir effect (FRE) (Brass 2016). Caneva's (1991) study focused on sherds curated at the British Museum. An assemblage she termed Dotted Wavy Line was dated to the sixth millennium based on superficially similar occurrences from other sites located in central Sudan.

A fundamental component of any chronological re-evaluation of Jebel Moya is its pottery. The first thorough review of the surviving pottery from Wellcome's excavations was carried out by Brass (2016). An attribute-based approach to ceramics and a thorough re-examination of burial data records also from Wellcome's excavations clarified some of the chronological sequence. Subsequently, this led to the first field season in 2017, which also included a re-examination of Phase 1 pottery curated at the National Museum in Khartoum (Brass et al., 2019, 2018c, 2018b). The latter was excavated by Wellcome's team and its examination greatly increased the range of recognised décor and its motor actions for the earliest known occupation of the site.

In total, 10 trenches were excavated in 2017 and 2019 (Brass et al., 2020, 2019, 2018a, 2018b) (Figure 2). Due to the lack of visual micro-stratigraphy, excavating in small $10 \mathrm{~cm}$ spits within the macro-geological strata provided a good measure of control (Brass et al., 2020). The trenches include a stone circle (T5), human burials (T3, 8, 9 and 10), surface collections (T7) and occupational remains (T1, 2, 4, 5). Trenches 1, 2, 4, 6 and 7 are not associated with burial activities, nor are there disturbances from burial activities or pits being dug. Trench 2 is currently excavated to a depth of $2.4 \mathrm{~m}$ well into the late $6^{\text {th }}$ millennium $\mathrm{BC}$ without hitting bedrock. Trench 2 has provided the best occupational sequence: any changes in material culture within a spit would be further detected by analysing the immediately preceding and succeeding spits. In line with the measures taken at Al Khiday (Salvatori et al. 2011), where occupational activity is apparent in wetter preservation conditions and therefore stratigraphy is more readily observable, micro-geomorphological analysis will be undertaken at Jebel Moya in forthcoming field seasons to fully flesh out site formation processes. However, the pottery and broad geological strata from Trench 2 in particular make it clear that there are discrete pottery assemblages per geological stratum, and as is outlined below there are changes in the lower spits of Assemblage 2 which have chronological implications for the site. 
The pottery was catalogued using standardised parameters to keep the information collected as clear and concise as possible on the dimensions, condition and specific features of the pottery. A total of 5733 sherds have been collected and analysed. Of these, 485 have decoration (Table 3). The majority of the sherds come from trenches 2 and 6. The pottery is divided into Assemblages $1-3$, and while the terminology is employed here for the sake of continuity, their variability is greatly expanded and re-defined.

\begin{tabular}{|c|c|c|c|c|c|c|c|}
\hline Spit & Trench 1 & Trench 2 & Trench 4 & Trench 6 & Trench 8 & Trench 9 & Trench 10 \\
\hline 1 & $\begin{array}{c}36 / 79 \\
\text { (Stratum A) }\end{array}$ & $\begin{array}{c}9 / 54 \\
\text { (Stratum A) }\end{array}$ & 0 (Stratum A) & 2/13 (Mixed) & $\begin{array}{c}10 / 50 \\
\text { (Stratum B) }\end{array}$ & $\begin{array}{c}0 / 3 \\
\text { (Stratum C) }\end{array}$ & $\begin{array}{c}\text { 20/83 } \\
\text { (Stratum } \\
\text { C) }\end{array}$ \\
\hline 2 & $18 / 34$ & $18 / 167$ & $10 / 20$ & $\begin{array}{c}11 / 64 \\
\text { (Mixed) }\end{array}$ & $1 / 27$ & & \\
\hline 3 & $\begin{array}{c}23 / 120 \\
\text { (Stratum B) }\end{array}$ & $25 / 241$ & $8 / 45$ & $\begin{array}{c}8 / 134 \\
\text { (Stratum B) }\end{array}$ & $\begin{array}{c}1 / 8(<27 \mathrm{~cm}) \\
2 / 17 \\
(>27 \mathrm{~cm}, \\
\text { Stratum C) }\end{array}$ & & \\
\hline 4 & $18 / 71$ & $15 / 283$ & $\begin{array}{c}5 / 35 \\
\text { (Stratum B) }\end{array}$ & $19 / 403$ & $6 / 41$ & & \\
\hline 5 & $2 / 17$ & $\begin{array}{c}10 / 139 \\
\text { (Stratum B) }\end{array}$ & $4 / 7$ & $\begin{array}{c}12 / 183 \\
\text { (Stratum C) }\end{array}$ & $1 / 1$ & & \\
\hline 6 & 0 & $9 / 190$ & $1 / 15$ & $14 / 182$ & & & \\
\hline 7 & $2 / 32$ & $8 / 170$ & $1 / 18$ & $6 / 114$ & & & \\
\hline 8 & $4 / 70$ & $6 / 559$ & $\begin{array}{c}8 / 84 \\
\text { (Stratum C) }\end{array}$ & $12 / 88$ & & & \\
\hline 9 & & $6 / 254$ & $2 / 16$ & & & & \\
\hline 10 & & $8 / 335$ & $1 / 6$ & & & & \\
\hline 11 & & $6 / 177$ & & & & & \\
\hline 12 & & $2 / 96$ & & & & & \\
\hline 13 & & 0 (Stratum C) & & & & & \\
\hline 14 & & $6 / 136$ & & & & & \\
\hline 15 & & $6 / 276$ & & & & & \\
\hline 16 & & $1 / 21$ & & & & & \\
\hline 17 & & $3 / 125$ & & & & & \\
\hline 18 & & $9 / 79$ & & & & & \\
\hline 19 & & $7 / 93$ & & & & & \\
\hline 20 & & $\begin{array}{c}33 / 126 \\
\text { (Stratum D) }\end{array}$ & & & & & \\
\hline 21 & & $\mathrm{~N} / \mathrm{A}$ & & & & & \\
\hline 22 & & $19 / 80$ & & & & & \\
\hline 23 & & $6 / 28$ & & & & & \\
\hline 24 & & $6 / 19$ & & & & & \\
\hline 25 & & 0 & & & & & \\
\hline 26 & & $2 / 5$ & & & & & \\
\hline & $103 / 423$ & $220 / 3653$ & $37 / 246$ & $84 / 1181$ & $21 / 144$ & $0 / 3$ & $20 / 83$ \\
\hline
\end{tabular}

Table 3. Decorated sherds and total number of sherds per spit per Trench. Total of 485 decorated 199 sherds out of 5733 overall sherds. 


\section{Results}

The presence and absence of attributes of the pottery sherds from the originating stratum (A - D) are compared here in order to further elucidate the nature of stratified variability present at Jebel Moya. This was accomplished by first quantifying the single occurrence of attributes in the different strata and by examining the co-occurrence of attributes across rim sherds on a per stratum basis. The following results are offered by Stratum, noting that Strata A and B contain Assemblage 3 sherds, Stratum C contains Assemblage 2 and Stratum D is Assemblage 1 (Figures 3-5). Because the assemblages have been defined as a whole so far and all our sherds broadly fall within those defined assemblages, we are using this broad level terminology. Our radiometric dates have allowed us to give a site chronological framework (Brass et al., 2019), but we need more dates to break down phases within those assemblages. Interestingly, the stratigraphy shows no changes in Assemblage 3 and some minor changes have been identified at the start of A2 which will be explored further as we excavate deeper.

\subsection{Surface treatments}

Burnishing and slipping are is present on $100 \%$ of the decorated sherds in Stratum A (Table 4), which is highly similar to the $93.94 \%$ and $100 \%$ respectively for the British Museum's (BM) sherds from the same stratum. In Trench 2's Stratum B, only 2 decorated sherds were unburnished and unslipped, with $100 \%$ respectively in Trench 4, which correlates with the BM's $92.11 \%$ and $93.42 \%$. It is in Trench 6 that a different trend emerges from Stratum B: Out of a total of 27 decorated sherds, $16(59.26 \%)$ are burnished and $13(48.15 \%)$ are slipped.

\begin{tabular}{|c|c|c|c|c|c|c|c|}
\hline Treatment & Spit & Trench 1 & Trench 2 & Trench 4 & Trench 6 & Trench 8 & $\begin{array}{c}\text { Trench } \\
10\end{array}$ \\
\hline \multirow[t]{17}{*}{ Burnished } & 1 & $\begin{array}{c}36(100 \%) \\
\text { (Stratum A) }\end{array}$ & $\begin{array}{c}9(100 \%) \\
\text { (Stratum A) }\end{array}$ & $\begin{array}{c}0(100 \%) \\
\text { (Stratum A) }\end{array}$ & $\begin{array}{l}2(100 \%) \\
\text { (Mixed) }\end{array}$ & $\begin{array}{c}10 \\
(100 \%)\end{array}$ & 0 \\
\hline & 2 & $18(100 \%)$ & $18(100 \%)$ & $10(100 \%)$ & $11(100 \%)$ & $1(100 \%)$ & \\
\hline & 3 & $\begin{array}{c}23(100 \%) \\
\text { (Stratum B) }\end{array}$ & 25 (100\%) & $8(100 \%)$ & $\begin{array}{c}8(100 \%) \\
\text { (Stratum B) }\end{array}$ & 0 & \\
\hline & 4 & $18(100 \%)$ & 15 (100\%) & $\begin{array}{c}5(100 \%) \\
\text { (Stratum B) }\end{array}$ & $8(42.1 \%)$ & 0 & \\
\hline & 5 & $0(0 \%)$ & $\begin{array}{c}9(90 \%) \\
\text { (Stratum B) }\end{array}$ & $4(100 \%)$ & $\begin{array}{l}2(16.67 \%) \\
\text { (Stratum C) }\end{array}$ & 0 & \\
\hline & 6 & $0(100 \%)$ & $8(88.9 \%)$ & $1(100 \%)$ & $6(42.86 \%)$ & & \\
\hline & 7 & $2(100 \%)$ & $8(100 \%)$ & $1(100 \%)$ & $1(16.67 \%)$ & & \\
\hline & 8 & $4(100 \%)$ & $6(100 \%)$ & $\begin{array}{c}8(100 \%) \\
\text { (Stratum C) }\end{array}$ & 0 & & \\
\hline & 9 & & $6(100 \%)$ & $2(100 \%)$ & & & \\
\hline & 10 & & $8(100 \%)$ & $1(100 \%)$ & & & \\
\hline & 11 & & $6(100 \%)$ & & & & \\
\hline & 12 & & $2(100 \%)$ & & & & \\
\hline & 13 & & $\begin{array}{c}0 \\
\text { (Stratum C) } \\
\end{array}$ & & & & \\
\hline & 14 & & $6(100 \%)$ & & & & \\
\hline & 15 & & $6(100 \%)$ & & & & \\
\hline & 16 & & $1(100 \%)$ & & & & \\
\hline & 17 & & $3(100 \%)$ & & & & \\
\hline
\end{tabular}




\begin{tabular}{|c|c|c|c|c|c|c|c|}
\hline & 18 & & 4 (44.44\%) & & & & \\
\hline & 19 & & $4(57.14 \%)$ & & & & \\
\hline & 20 & & $\begin{array}{c}3(100 \%) \\
\text { (Stratum D) }\end{array}$ & & & & \\
\hline & 21 & & 0 & & & & \\
\hline & 22 & & 19 (100\%) & & & & \\
\hline & 23 & & 0 & & & & \\
\hline & 24 & & 0 & & & & \\
\hline & 25 & & 0 & & & & \\
\hline & 26 & & 0 & & & & \\
\hline \multirow[t]{26}{*}{ Slipping } & 1 & $\begin{array}{c}36(100 \%) \\
\text { (Stratum A) }\end{array}$ & $\begin{array}{c}9(100 \%) \\
\text { (Stratum A) }\end{array}$ & $\begin{array}{c}0(100 \%) \\
\text { (Stratum A) }\end{array}$ & $\begin{array}{l}2(100 \%) \\
\text { (Mixed) }\end{array}$ & $\begin{array}{c}10 \\
(100 \%) \\
\end{array}$ & 0 \\
\hline & 2 & $18(100 \%)$ & $18(100 \%)$ & $10(100 \%)$ & $11(100 \%)$ & $1(100 \%)$ & \\
\hline & 3 & $\begin{array}{c}23(100 \%) \\
\text { (Stratum B) }\end{array}$ & 25 (100\%) & $8(100 \%)$ & $\begin{array}{c}8(100 \%) \\
\text { (Stratum B) }\end{array}$ & $\begin{array}{c}1 \\
(33.33 \%) \\
\end{array}$ & \\
\hline & 4 & $18(100 \%)$ & 15 (100\%) & $\begin{array}{c}5(100 \%) \\
\text { (Stratum B) }\end{array}$ & $5(26.32 \%)$ & 0 & \\
\hline & 5 & $2(100 \%)$ & $\begin{array}{c}9(90 \%) \\
\text { (Stratum B) }\end{array}$ & $4(100 \%)$ & $\begin{array}{c}0 \\
\text { (Stratum C) }\end{array}$ & 0 & \\
\hline & 6 & 0 (100\%) & 8 (88.9\%) & $1(100 \%)$ & 0 & & \\
\hline & 7 & $2(100 \%)$ & $8(100 \%)$ & $1(100 \%)$ & $1(16.67 \%)$ & & \\
\hline & 8 & $4(100 \%)$ & $6(100 \%)$ & $\begin{array}{c}8(100 \%) \\
\text { (Stratum C) }\end{array}$ & 0 & & \\
\hline & 9 & & $6(100 \%)$ & $2(100 \%)$ & & & \\
\hline & 10 & & $8(100 \%)$ & $1(100 \%)$ & & & \\
\hline & 11 & & $6(100 \%)$ & & & & \\
\hline & 12 & & $2(100 \%)$ & & & & \\
\hline & 13 & & $\begin{array}{c}0 \\
\text { (Stratum C) }\end{array}$ & & & & \\
\hline & 14 & & $6(100 \%)$ & & & & \\
\hline & 15 & & $6(100 \%)$ & & & & \\
\hline & 16 & & $1(100 \%)$ & & & & \\
\hline & 17 & & $3(100 \%)$ & & & & \\
\hline & 18 & & $3(33.33 \%)$ & & & & \\
\hline & 19 & & $2(28.57 \%)$ & & & & \\
\hline & 20 & & $\begin{array}{c}0 \\
\text { (Stratum D) }\end{array}$ & & & & \\
\hline & 21 & & $\mathrm{~N} / \mathrm{A}$ & & & & \\
\hline & 22 & & 0 & & & & \\
\hline & 23 & & 0 & & & & \\
\hline & 24 & & 0 & & & & \\
\hline & 25 & & 0 & & & & \\
\hline & 26 & & 0 & & & & \\
\hline
\end{tabular}

223 Table 4. Surface treatments across the strata and their respective percentage out of all decorated 224 sherds within each stratum. 
In Stratum C in Trench 2, it is only the sherds from the lowermost spits (18 and 19) which are not $100 \%$ burnished: their rate drops to $44.44 \%$ and $57.14 \%$ respectively. The sherds from spits 18 and 19 also have equivalent drop for slipping, down to $33.33 \%$ and $28.57 \%$.

In Trench 6, all the decorated sherds from Statum C are burnished and slipped. While there is a mixture of slipped and unslipped decorated sherds in Trench 8, none of the decorated sherds in the adjacent Trench 10 are slipped.

The Stratum D sherds from Trench 2's spits 20 and 21 are all burnished, but the decorated sherds from the remaining excavated Stratum D spits are unburnished. All the sherds though are unslipped. The presence of some burnishing contrasts to the BM's late 6th millennium sherds which are neither burnished nor slipped.

\subsection{Rim forms and décor}

The BM collection exhibited four types of rims: straight (simple) thin, straight thin, everted and everted rolled (Brass, 2016: Table 3.4). This variety is reiterated and extended to include flat, straight rolled, thin in-sloping (not fully inverted), thick in-sloping and thin everted (Table 5). For Stratum A, straight thin rims predominate (45.95\%), as they do in the BM collection $(88.46 \%)$, although there is greater abundance of straight thick rims $(28.38 \%$ versus $4.81 \%)$. However, thin everted rims make their first appearance (5.41\%). In Stratum B, as in the BM collection, thin straight rims continue dominating (46.3\%) and thin everted rims increase in preponderance $(15.74 \%)$ while there is one example of a rolled straight rim.

\begin{tabular}{|c|c|c|c|c|}
\hline Rim form & Stratum A & Stratum B & Stratum C & Stratum D \\
\hline Flat & & & $3(3.8 \%)$ & \\
\hline $\mathbf{R E}$ & 9 (12.16\%) & 17 (15.74\%) & $7(8.88 \%)$ & \\
\hline RER & & & $1(1.25 \%)$ & \\
\hline RS & & $1(0.93 \%)$ & & \\
\hline RSTK & $21(28.38 \%)$ & $15(13.9 \%)$ & $24(30 \%)$ & $3(27.27 \%)$ \\
\hline RSTN & 34 (45.95\%) & $50(46.3 \%)$ & $28(35 \%)$ & $8(72.73 \%)$ \\
\hline TE & $4(5.41 \%)$ & $17(15.74 \%)$ & $8(10 \%)$ & \\
\hline THI & $1(1.35 \%)$ & & $2(2.5 \%)$ & \\
\hline TI & $5(6.76 \%)$ & $6(5.56 \%)$ & $4(5 \%)$ & \\
\hline TU & & $2(1.85 \%)$ & & \\
\hline Too small & & & $2(2.5 \%)$ & \\
\hline Total & 74 & 108 & 80 & 11 \\
\hline
\end{tabular}

Table 5. The different (decorated and undecorated) rim forms present across the strata and their respective percentage within each stratum

Rim codes

RE: Thick everted

RER: Everted rolled

RS: Rolled, straight

RSTK: Straight thick
TE: Thin everted

THI: Thick in-sloping

TI: Thin in-sloping

TU: Thick, unknown 


\section{RSTN: Straight thin}

Stratum C has the greater variety of rims. The two dominant types are thick straight $(30.38 \%)$ and thin straight $(35.44 \%)$. In the BM collection, there were more thick everted and thick everted rolled sherds (17 versus the currently excavated 8 ). This variety is greatly diminished for Stratum $\mathrm{D}$ to just thick and thin straight rims, but it is too soon to tell if this is an artefact of lesser occupation density.

The largest variety of rim forms (9) are present in Stratum C, compared with seven in B and six in A. Only two rim forms were found amongst the Stratum D sherds, but more late 6th millennium BC layers need to be excavated. While the rims from strata A and B correspond with Assemblage 1 from the British Museum, the Stratum $\mathrm{C}$ rims expand the previously known range of Assemblage 2 (Brass 2016: Table 3.4). Although thin straight rims form the majority in both strata B and C with $46.3 \%$ and $35 \%$ respectively, there is also a large proportion of thick straight rims $(30 \%)$ for Stratum C while the second most prevalent rim form for Stratum B are thin everted rims (15.74\%). Thick everted rims are present in both strata B and C, but the type of thick everted rolled rims characteristic of Assemblage 2 from the British Museum are present in Stratum C.

Only one type of décor and tool was used per rim from all strata (Table 6). Décor is present on more rims from strata A and B than C. Stylus motor actions overwhelmingly dominate Stratum A rim decors, with incised lines the majority at $42.37 \%$. There is little comb stamping. In Stratum B, while incised lines still dominate at $41.38 \%$, there is an increase in the variety and numbers of comb-stamping. Stamped dotted lines account for 17.24\%. In Stratum C, there is a more even distribution: dragged comb at $15.56 \%$, incised lines at $26.67 \%$ and dragged comb angular lines at $13.33 \%$, while packed zigzag dots, rocker stamped comb incised banded lines, dotted stamped triangles, Dragged comb angular lines meeting to form chevron impressions, banded incised lines and incised lines with curvatures make their first appearance. In Stratum D, the late Mesolithic rims, there are 6 instances of stamped dotted lines, 2 APS (alternately pivoting stamp) paired line fans (one occurrence in Stratum B), one incised line and one incised chevrons.

\begin{tabular}{|c|c|c|c|c|c|}
\hline Tool & Motor action & Stratum A & Stratum B & Stratum C & Stratum D \\
\hline \multicolumn{6}{|l|}{ Nail } \\
\hline & $\mathrm{N}$ & 1 (1.69\%) & & & \\
\hline \multicolumn{6}{|l|}{ Stylus } \\
\hline & IC & $11(18.64 \%)$ & $9(10.34 \%)$ & $6(13.33 \%)$ & $1(10 \%)$ \\
\hline & $\mathrm{ICHT}$ & $6(10.17 \%)$ & $6(6.9 \%)$ & $3(6.67 \%)$ & \\
\hline & $\mathrm{ICL}$ & 1 (1.69\%) & & & \\
\hline & ILS & 25 (42.37\%) & $36(41.38 \%)$ & $12(26.67 \%)$ & $1(10 \%)$ \\
\hline & ILSB & & & $1(2.22 \%)$ & \\
\hline & ILSC & & & $2(4.44 \%)$ & \\
\hline \multicolumn{6}{|l|}{ Comb } \\
\hline & APS-PF & & $1(1.15 \%)$ & & $2(20 \%)$ \\
\hline & DC & 1 (1.69\%) & $3(3.45 \%)$ & 7 (15.56\%) & \\
\hline
\end{tabular}




\begin{tabular}{|c|c|c|c|c|c|}
\hline & DCAL & & $3(3.45 \%)$ & $6(13.33 \%)$ & \\
\hline & DCAL-C & & & $1(2.22 \%)$ & \\
\hline & $\mathrm{DCH}$ & & $1(1.15 \%)$ & & \\
\hline & $\mathrm{SCHT}$ & $1(1.69 \%)$ & 1 (1.15\%) & & \\
\hline & $\mathrm{SL}$ & & & $2(4.44 \%)$ & \\
\hline & SL-BLD & 1 (1.69\%) & & & \\
\hline & SL-D & $7(1.69 \%)$ & 15 (17.24\%) & & $6(60 \%)$ \\
\hline & SL-PD & & $2(2.3 \%)$ & & \\
\hline & SL-PS & 1 (1.69\%) & & & \\
\hline & SL-PZD & & & $1(2.22 \%)$ & \\
\hline & SL-RILS & & & $1(2.22 \%)$ & \\
\hline & SL-S & $1(1.69 \%)$ & $4(4.6 \%)$ & $1(2.22 \%)$ & \\
\hline & SL-TT & & $1(1.15 \%)$ & & \\
\hline & SL-US & $3(5.08 \%)$ & $2(2.3 \%)$ & $1(2.22 \%)$ & \\
\hline & SS & & 1 (1.15\%) & & \\
\hline & ST-D & & & $1(2.22 \%)$ & \\
\hline & $U$ & & 1 (1.15\%) & & \\
\hline \multicolumn{6}{|l|}{ Clay } \\
\hline & $W C$ & & 1 (1.15\%) & & \\
\hline Total & & 59 & 87 & 45 & 10 \\
\hline
\end{tabular}

Table 6. Rim sherds: Single attribute occurrences of decor tools and the corresponding motor actions. The percentage is of total decorated rims per stratum.

In Stratum A, the majority $(50.85 \%)$ of décor was on thin straight rims and the highest occurrences were stylus-incised channels (below the rim) and lines (8.48\%) (Table 7). The décor was made using fingernails, styluses and combs. In Stratum B, décor was also predominantly on thin straight rims $(48.39 \%)$. The occurrence of incised lines increased to $20.43 \%$, while incised channels increased to $20.43 \%$, while incised channels remained steady at $6.45 \%$ for the same rim type. The variety of motor actions increased in Stratum B (Table 8). While there were no nails used as an implement, the actions of styluses and combs now included incised angular lines at opposing angles (banded), incised cross-hatching formed by overlapping chevrons, incised lines with curvature, paired fan lines (APS), dragged comb angular lines, dragged comb vertical lines, plain dashed comb-stamped lines comb-stamped (triangular toothed) lines and stylus stabs. The packed square-toothed comb lines from Stratum A are not present in Stratum B.

\begin{tabular}{|l|l|l|l|l|l|l|l|}
\hline & & RE & RSTK & RSTN & TE & THI & TI \\
\hline Nails & & & & & & & \\
\hline & N & & & $1(1.69 \%)$ & & & \\
\hline Stylus & & & & & & & \\
\hline & IC & $2(3.39 \%)$ & $2(3.39 \%)$ & $5(8.48 \%)$ & $1(1.69 \%)$ & & $1(1.69 \%)$ \\
\hline & ICHT & & $2(3.39 \%)$ & 4 & & & \\
\hline & ICL & & & $1(1.69 \%)$ & & & \\
\hline
\end{tabular}




\begin{tabular}{|c|c|c|c|c|c|c|c|}
\hline & ILS & 1 (1.69\%) & $6(10.17)$ & $\begin{array}{l}11 \\
(18.64 \%)\end{array}$ & $3(5.08 \%)$ & $1(1.69 \%)$ & $3(5.08 \%)$ \\
\hline \multicolumn{8}{|l|}{ Comb } \\
\hline & DC & & & & & & 1 (1.69\%) \\
\hline & $\mathrm{SCHT}$ & & & & 1 (1.69\%) & & \\
\hline & SL-BLD & & 1 (1.69\%) & & & & \\
\hline & SL-D & & 2 (3.39\%) & 5 (8.48\%) & & & \\
\hline & SL-PS & & & 1 (1.69\%) & & & \\
\hline & SL-S & & 1 (1.69\%) & & & & \\
\hline & SL-US & & & 2 (3.39\%) & 1 (1.69\%) & & \\
\hline Total & & $3(5.08 \%)$ & $\begin{array}{l}14 \\
\text { (23.73\%) }\end{array}$ & $\begin{array}{l}30 \\
(50.85 \%)\end{array}$ & $\begin{array}{l}6 \\
(10.17 \%)\end{array}$ & 1 (1.69\%) & $5(8.48 \%)$ \\
\hline
\end{tabular}

Table 7. Co-occurrences of rim types and motor actions for Stratum A. The percentage is of total decorated rims (59).

305

\begin{tabular}{|c|c|c|c|c|c|c|c|c|}
\hline & & RE & RSTK & RSTN & $\mathrm{TE}$ & THI & $\mathrm{TI}$ & TU \\
\hline \multicolumn{9}{|l|}{ Stylus } \\
\hline & IALO & & & $1(1.08 \%)$ & & & & \\
\hline & IC & $1(1.08 \%)$ & $1(1.08 \%)$ & $6(6.45 \%)$ & $2(2.15 \%)$ & $1(1.08 \%)$ & & \\
\hline & $\mathrm{ICCHT}$ & & $1(1.08 \%)$ & & & & & \\
\hline & ICHT & & $3(3.23 \%)$ & 3 (3.23\%) & & & & 1 (1.08\%) \\
\hline & ILS & $7(7.53 \%)$ & $5(5.38 \%)$ & $\begin{array}{l}19 \\
(20.43 \%)\end{array}$ & $6(6.45 \%)$ & & $1(1.08 \%)$ & $2(2.15 \%)$ \\
\hline & ILSC & & 2 (2.15\%) & & & & & \\
\hline \multicolumn{9}{|l|}{ Comb } \\
\hline & APS-PF & & $1(1.08 \%)$ & & & & & \\
\hline & $\mathrm{DC}$ & & & $1(1.08 \%)$ & $1(1.08 \%)$ & & & \\
\hline & DCAL & & & $2(2.15 \%)$ & & & & \\
\hline & DCVL & & $1(1.08 \%)$ & & & & & \\
\hline & SL & & $1(1.08 \%)$ & & & & & \\
\hline & SL-D & $1(1.08 \%)$ & $2(2.15 \%)$ & $8(8.6 \%)$ & & & $1(1.08 \%)$ & \\
\hline & SL-DP & & & & & & 1 (1.08\%) & \\
\hline & SL-PD & & $1(1.08 \%)$ & $1(1.08 \%)$ & & & & \\
\hline & SL-S & & $1(1.08 \%)$ & $3(3.23 \%)$ & & & & \\
\hline & SL-TT & & & & $1(1.08 \%)$ & & & \\
\hline & SL-US & & $2(2.15 \%)$ & & & & & \\
\hline & SS & & & $1(1.08 \%)$ & & & & \\
\hline \multicolumn{9}{|c|}{ Too worn } \\
\hline & U & 1 (1.08\%) & & & & & & \\
\hline Total & & $\begin{array}{l}10 \\
(10.75 \%)\end{array}$ & $\begin{array}{l}21 \\
(22.58 \%)\end{array}$ & $\begin{array}{l}45 \\
\text { (48.39\%) }\end{array}$ & $\begin{array}{l}10 \\
(10.75 \%)\end{array}$ & $1(1.08 \%)$ & $3(3.23 \%)$ & $3(3.23 \%)$ \\
\hline
\end{tabular}


The co-occurrences were more variable in Stratum C (Table 9): 37.78\% thin straight and 26.27\% thick straight rims, while there is the first occurrence of the BM's Assemblage 2 diagnostic thick everted, rolled rims. The majority of the Assemblage 2 décor was made using a comb and there were distinctly unique motor actions: banded stylus-incised lines, dragged comb angular lines meeting to form chevron impressions, comb-stamped lines of packed zigzag dots, rockerstamped comb inside incised banded lines and dotted comb-stamped lines. Dragged comb chevrons had the highest occurrence and where present on thick everted rolled $(2.22 \%)$ and thin straight rims $(8.89 \%)$.

\begin{tabular}{|c|c|c|c|c|c|c|c|c|c|c|}
\hline & & RE & RER & RSTK & RSTN & TE & THI & $\mathrm{TI}$ & $\begin{array}{l}\begin{array}{l}\text { Too } \\
\text { small }\end{array} \\
\end{array}$ & TU \\
\hline \multicolumn{11}{|l|}{ Stylus } \\
\hline & IC & $\begin{array}{l}1 \\
(2.22 \%) \\
\end{array}$ & & 2 & $\begin{array}{l}1 \\
(2.22 \%) \\
\end{array}$ & $\begin{array}{l}1 \\
(2.22 \%) \\
\end{array}$ & $\begin{array}{l}1 \\
(2.22 \%) \\
\end{array}$ & & & \\
\hline & $\mathrm{ICHT}$ & & & $\begin{array}{l}1 \\
(2.22 \%)\end{array}$ & $\begin{array}{l}1 \\
(2.22 \%)\end{array}$ & & & & & $\begin{array}{l}1 \\
(2.22 \%)\end{array}$ \\
\hline & ILS & $\begin{array}{l}1 \\
(2.22 \%) \\
\end{array}$ & & $\begin{array}{l}3 \\
(6.67 \%)\end{array}$ & 5 & $\begin{array}{l}3 \\
(6.67 \%)\end{array}$ & & & & \\
\hline & ILSB & & & & $\begin{array}{l}1 \\
(2.22 \%)\end{array}$ & & & & & \\
\hline & ILSC & & & $\begin{array}{l}2 \\
(4.44 \%) \\
\end{array}$ & & & & & & \\
\hline \multicolumn{11}{|l|}{ Comb } \\
\hline & $\mathrm{DC}$ & & $\begin{array}{l}1 \\
(2.22 \%)\end{array}$ & & $\begin{array}{l}4 \\
(8.89 \%)\end{array}$ & & & $\begin{array}{l}2 \\
(4.44 \%)\end{array}$ & & \\
\hline & DCAL & $\begin{array}{l}1 \\
(2.22 \%) \\
\end{array}$ & & $\begin{array}{l}2 \\
(4.44 \%)\end{array}$ & $\begin{array}{l}2 \\
(4.44 \%)\end{array}$ & & & $\begin{array}{l}1 \\
(2.22 \%)\end{array}$ & & \\
\hline & DCAL-C & & & $\begin{array}{l}1 \\
(2.22 \%)\end{array}$ & & & & & & \\
\hline & $S L$ & & & $\begin{array}{l}1 \\
(2.22 \%) \\
\end{array}$ & $\begin{array}{l}1 \\
(2.22 \%) \\
\end{array}$ & & & & & \\
\hline & SL-PZD & & & & & & & $\begin{array}{l}1 \\
(2.22 \%)\end{array}$ & & \\
\hline & SL-RILS & & & & & & & & $\begin{array}{l}1 \\
(2.22 \%) \\
\end{array}$ & \\
\hline & SL-S & & & & & & & $\begin{array}{l}1 \\
(2.22 \%)\end{array}$ & & \\
\hline & SL-US & & & & $\begin{array}{l}1 \\
(2.22 \%)\end{array}$ & & & & & \\
\hline & ST-D & & & & $\begin{array}{l}1 \\
(2.22 \%)\end{array}$ & & & & & \\
\hline & Total & $\begin{array}{l}3 \\
(6.67 \%)\end{array}$ & $\begin{array}{l}1 \\
(2.22 \%)\end{array}$ & $\begin{array}{l}12 \\
(26.67 \% \\
)\end{array}$ & $\begin{array}{l}17 \\
(37.78 \% \\
)\end{array}$ & $\begin{array}{l}4 \\
(8.89 \%)\end{array}$ & $\begin{array}{l}1 \\
(2.22 \%)\end{array}$ & $\begin{array}{l}5 \\
(11.11 \% \\
)^{2}\end{array}$ & $\begin{array}{l}1 \\
(2.22 \%)\end{array}$ & $\begin{array}{l}1 \\
(2.22 \%)\end{array}$ \\
\hline
\end{tabular}
decorated rims (45). 
321 By contrast, there are currently very few Assemblage 1 rims (Table 10). There are just two types:

322 thin and thick straight rims with limited stylus and comb décor. However, an occurrence of APS

323 paired dotted lines was recorded.

324

\begin{tabular}{|l|l|l|l|}
\hline & & RSTK & RSTN \\
\hline Stylus & & & \\
\hline & IC & $1(10 \%)$ & \\
\hline & ILS & $1(10 \%)$ & \\
\hline Comb & & & \\
\hline & APS-PL & $1(10 \%)$ & $1(10 \%)$ \\
\hline & SL-D & & $6(60 \%)$ \\
\hline Total & & $\mathbf{3}$ & $\mathbf{7}$ \\
\hline
\end{tabular}

325 Table 10. Co-occurrences of rim types and motor actions for Stratum D. The percentage is of 326 total decorated rims (10).

327

\section{$328 \quad 3.3$ Body sherds and décor}

329 There are 518 body sherds with décor across all the strata (Table 11). These show a greater range 330 of motor actions and types of décor than the rims. Frequently, more than one type of décor was 331 present. APS is present in both strata $\mathrm{C}$ and D. Stratum $\mathrm{C}$ has the following APS décor: standard 332 APS, curved lines, paired fan lines, smocking and double-pronged wavy lines. Smocking is also 333 present at Shaqadud Midden in the Central Sudan, north-western Butana Plain (Brass et al., 334 2018c).

335

\begin{tabular}{|l|c|c|c|c|}
\hline \multicolumn{1}{|c|}{ Décor } & Stratum D & Stratum C & Strata A and B & Total \\
\hline ACU & & & $1(0.37 \%)$ & 1 \\
\hline APS & & $9(5.52 \%)$ & & 1 \\
\hline APS-CL & & $1(0.61 \%)$ & & 2 \\
\hline APS-PF & & $2(1.23 \%)$ & & 5 \\
\hline APS-PL & $3(3.66 \%)$ & & $2(0.73 \%)$ & 1 \\
\hline APS-SM & & $1(0.61 \%)$ & & 1 \\
\hline APS-WL & & $1(0.61 \%)$ & & 1 \\
\hline BDC & & $1(0.61 \%)$ & & 5 \\
\hline CEI & & & $1(1.83 \%)$ & 2 \\
\hline CER & & & & 1 \\
\hline CF & $2(2.44 \%)$ & $1(0.61 \%)$ & & 7 \\
\hline CWI & & $4(2.45 \%)$ & & 7 \\
\hline DCAL & $3(3.66 \%)$ & $6(3.68 \%)$ & & 3 \\
\hline DCH & $1(1.22 \%)$ & $3(1.84 \%)$ & & 1 \\
\hline DCH-ILS & & $1(0.61 \%)$ & & 1 \\
\hline DCJ & & $1(0.61 \%)$ & & 1 \\
\hline DCV & $1(1.22 \%)$ & & & \\
\hline DLC & & & & \\
\hline
\end{tabular}




\begin{tabular}{|c|c|c|c|c|}
\hline $\mathrm{F}$ & & & $1(0.37 \%)$ & 1 \\
\hline FS-R & & $1(0.61 \%)$ & & 1 \\
\hline FT & 3 (3.66\%) & & & 3 \\
\hline HR & & 1 (0.61\%) & & 1 \\
\hline IALO & & $2(0.61 \%)$ & & 2 \\
\hline IC & 1 (1.22\%) & 11 (6.75\%) & 26 (9.52\%) & 38 \\
\hline ICCHT & & $2(1.23 \%)$ & & 2 \\
\hline $\mathrm{ICHT}$ & & $11(6.75 \%)$ & $6(2.2 \%)$ & 17 \\
\hline ICJ & & $1(0.61 \%)$ & & 1 \\
\hline ICL & & 21 (12.88\%) & $21(7.69 \%)$ & 42 \\
\hline IDL & & & $2(0.73 \%)$ & 2 \\
\hline IF & $1(1.22 \%)$ & & & 1 \\
\hline IG & & & $1(0.37 \%)$ & 1 \\
\hline $\mathrm{IH}$ & & $1(0.61 \%)$ & $2(0.73 \%)$ & 3 \\
\hline IILS & & & $4(1.47 \%)$ & 4 \\
\hline ILS & 1 (1.22\%) & $22(13.5 \%)$ & 64 (23.44\%) & 87 \\
\hline ILSB & & & $3(1.1 \%)$ & 3 \\
\hline ILSB-ILS & & $2(1.23 \%)$ & & 2 \\
\hline IQ & & & $2(0.73 \%)$ & 2 \\
\hline $\mathrm{N}$ & & 2 (1.23\%) & $4(1.47 \%)$ & 6 \\
\hline $\mathrm{N}$-SLS & & 1 (0.61\%) & & 1 \\
\hline $\mathrm{P}$ & 1 (1.22\%) & & $14(5.13 \%)$ & 15 \\
\hline PS & & $1(0.61 \%)$ & & 1 \\
\hline$R-C$ & $7(8.54 \%)$ & & & 7 \\
\hline R-TC & $5(6.1 \%)$ & & & 5 \\
\hline SC-D & $1(1.22 \%)$ & & $1(0.37 \%)$ & 2 \\
\hline $\mathrm{SCHL}$ & & $3(1.84 \%)$ & 4 (1.47\%) & 7 \\
\hline SCHL-US & & $1(0.61 \%)$ & & 1 \\
\hline SD & 1 (1.22\%) & & & 1 \\
\hline SDCHT & & & $1(0.37 \%)$ & 1 \\
\hline SIBDL & & & $1(0.37 \%)$ & 1 \\
\hline SL & $12(14.63 \%)$ & $2(1.23 \%)$ & $5(1.83 \%)$ & 19 \\
\hline SL-BA & $3(3.66 \%)$ & $1(0.61 \%)$ & & 4 \\
\hline SL-BASL & 1 (1.22\%) & & & 1 \\
\hline SL-BCD & 4 (2.44\%) & $1(0.61 \%)$ & & 5 \\
\hline SL-BD & & & $3(1.1 \%)$ & 3 \\
\hline SL-BDT & & & $2(0.73 \%)$ & 2 \\
\hline SL-BLD & & & $2(0.73 \%)$ & 2 \\
\hline SL-CBV & & $1(0.61 \%)$ & $1(0.37 \%)$ & 2 \\
\hline SL-D & $8(9.76 \%)$ & $17(10.43 \%)$ & $51(18.68 \%)$ & 76 \\
\hline SL-DD & & & $1(0.37 \%)$ & 1 \\
\hline
\end{tabular}




\begin{tabular}{|c|c|c|c|c|}
\hline SL-DP & $1(1.22 \%)$ & 2 (1.23\%) & & 3 \\
\hline SL-DR & $6(7.32 \%)$ & $4(2.45 \%)$ & & 10 \\
\hline SL-PD & & 2 (1.23\%) & $2(0.73 \%)$ & 4 \\
\hline SL-PZD & & $1(0.61 \%)$ & & 1 \\
\hline SL-S & $3(3.66 \%)$ & & $14(5.13 \%)$ & 17 \\
\hline SL-SD & & & $1(0.37 \%)$ & 1 \\
\hline SL-TTD & $6(7.32 \%)$ & & & 6 \\
\hline SL-UBD & & & $2(0.73 \%)$ & 2 \\
\hline SL-UCPD & $1(1.22 \%)$ & & & 1 \\
\hline SL-US & $3(3.66 \%)$ & 9 (5.52\%) & $9(3.3 \%)$ & 21 \\
\hline SL-W & & & $2(0.73 \%)$ & 2 \\
\hline$S Q$ & & & $2(0.73 \%)$ & 2 \\
\hline SQ-U & & & $1(0.37 \%)$ & 1 \\
\hline SSH & $1(1.22 \%)$ & $1(0.61 \%)$ & & 2 \\
\hline SSL & & & $2(0.73 \%)$ & 2 \\
\hline SSL-C & & $2(1.23 \%)$ & & 2 \\
\hline SSTB & & $1(0.61 \%)$ & $1(0.37 \%)$ & 2 \\
\hline ST-D & $1(1.22 \%)$ & & $1(0.37 \%)$ & 2 \\
\hline TD & & $1(0.61 \%)$ & & 1 \\
\hline TD-C & $1(1.22 \%)$ & & & 1 \\
\hline$U$ & & & $2(0.73 \%)$ & 2 \\
\hline WC & & $4(2.45 \%)$ & $3(1.1 \%)$ & 7 \\
\hline Total & 82 & 163 & 273 & 518 \\
\hline
\end{tabular}

336 Table 11. Body sherds by strata: Single attribute occurrences of decor tools and their 337 corresponding motor action. The percentage is of the total occurrences of motor actions per 338 assemblage.

339

\begin{tabular}{|l|l|l|l|}
\hline$A C U$ & $\begin{array}{l}\text { Applied clay, unknown } \\
\text { motif }\end{array}$ & $R-T C$ & Roulette, twisted cord \\
\hline$A P S-C L$ & APS, curved lines & $S C-D$ & Stamped chevrons, dotted \\
\hline$A P S-P L$ & APS, dotted paired lines & $S C H L$ & Stamped channel \\
\hline$A P S-P F$ & APS, paired fan lines & $S C H L-U S$ & $\begin{array}{l}\text { Stamped channel, unevenly } \\
\text { serrated }\end{array}$ \\
\hline$A P S-S M$ & APS, smocking & $S C H T$ & Stamped cross hatching \\
\hline$A P S-W L$ & $\begin{array}{l}\text { APS, double-pronged wavy } \\
\text { lines }\end{array}$ & $S D$ & Stamped dots \\
\hline$B D C$ & $\begin{array}{l}\text { Branch dragged channel } \\
C E I\end{array}$ & $S D C H T$ & $\begin{array}{l}\text { Stamped dashed cross } \\
\text { hatching }\end{array}$ \\
\hline$C E R$ & Cord wrapped element & $S I B D L$ & $\begin{array}{l}\text { Stamped infilled banded } \\
\text { dotted line(s) }\end{array}$ \\
\hline$C F$ & $\begin{array}{l}\text { Cord wrapped element } \\
\text { rolled lines }\end{array}$ & $S L$ & $\begin{array}{l}\text { Stamped lines } \\
\text { (indeterminate) }\end{array}$ \\
\hline$C W I$ & Cord, flipped & $S L-B A S L$ & $\begin{array}{l}\text { Unevenly serrated comb- } \\
\text { stamped angular lines, } \\
\text { banded by stylus-incised } \\
\text { horizontal lines }\end{array}$ \\
\hline
\end{tabular}




\begin{tabular}{|c|c|c|c|}
\hline$\overline{D C}$ & Dragged chevrons & $S L-B C D$ & $\begin{array}{l}\text { Stamped lines, banded by } \\
\text { comb-dragged lines }\end{array}$ \\
\hline $\mathrm{DCH}$ & Dragged channel & $S L-B D$ & $\begin{array}{l}\text { Stamped lines, banded } \\
\text { squares }\end{array}$ \\
\hline$D C H-I L S$ & $\begin{array}{l}\text { Dragged channel, infilled } \\
\text { with stylus-incised lines }\end{array}$ & $S L-B D T$ & Stamped lines, banded dots \\
\hline$D C J$ & Dragged chevrons, joined & $S L-B L D$ & $\begin{array}{l}\text { Stamped lines, banded lines } \\
\text { of dashes }\end{array}$ \\
\hline$D C A L$ & Dragged comb angular lines & $S L-B P D$ & $\begin{array}{l}\text { Stamped lines, banded } \\
\text { packed dots }\end{array}$ \\
\hline$D C A L-C$ & $\begin{array}{l}\text { Dragged comb angular lines } \\
\text { meeting to form chevron } \\
\text { impressions }\end{array}$ & $S L-C B V$ & $\begin{array}{l}\text { Stamped lines: curved, } \\
\text { banded, vertical }\end{array}$ \\
\hline$D C L$ & Dragged comb lines & $S L-D$ & Stamped lines, dotted \\
\hline$\overline{D C V}$ & $\begin{array}{l}\text { Dragged comb V-shape } \\
\text { lines (herringbone) }\end{array}$ & $S L-D D$ & $\begin{array}{l}\text { Stamped line, dotted } \\
\text { droplets }\end{array}$ \\
\hline$D C V L$ & Dragged comb vertical lines & $S L-D P$ & $\begin{array}{l}\text { Stamped lines, dotted } \\
\text { packed }\end{array}$ \\
\hline$F$ & Fillets & $S L-D R$ & $\begin{array}{l}\text { Stamped lines, dotted } \\
\text { rocker }\end{array}$ \\
\hline$F T$ & Fingertip impressions & $S L-P D$ & Stamped lines, plain dashed \\
\hline$F S-R$ & Fish spine, rolled & $S L-P S$ & $\begin{array}{l}\text { Stamped lines, packed } \\
\text { squares }\end{array}$ \\
\hline$H R$ & Hollow reed & $S L-P Z D$ & $\begin{array}{l}\text { Stamped lines, packed } \\
\text { zigzag dots }\end{array}$ \\
\hline$I A L O$ & $\begin{array}{l}\text { Incised angular lines at } \\
\text { opposing angles, banded }\end{array}$ & SL-RILS & $\begin{array}{l}\text { Rocker comb inside incised } \\
\text { banded lines }\end{array}$ \\
\hline$I C$ & Incised chevrons & $S L-S$ & Stamped lines, square \\
\hline ICCHT & $\begin{array}{l}\text { Incised cross-hatching } \\
\text { formed by overlapping } \\
\text { chevrons }\end{array}$ & $S L-S D$ & $\begin{array}{l}\text { Stamped lines, square and } \\
\text { dotted in same line }\end{array}$ \\
\hline$I C H T$ & Incised cross hatching & $S L-T T$ & $\begin{array}{l}\text { Stamped lines, triangular } \\
\text { toothed }\end{array}$ \\
\hline$I C J$ & Incised chevrons, joined & $S L-T T D$ & $\begin{array}{l}\text { Stamped line(s), dotted } \\
\text { two-toothed (not APS) }\end{array}$ \\
\hline$I C L$ & Incised channel(s) & $S L-U B D$ & $\begin{array}{l}\text { Stamped banded lines, } \\
\text { unevenly serrated dots }\end{array}$ \\
\hline$I D L$ & Incised dashed line(s) & $S L-U C P D$ & $\begin{array}{l}\text { Stamped lines, unevenly } \\
\text { serrated continuous packed } \\
\text { dashes }\end{array}$ \\
\hline$I F$ & Incised fan & $S L-U S$ & $\begin{array}{l}\text { Stamped lines, unevenly } \\
\text { serrated dots }\end{array}$ \\
\hline$I G$ & Incised grooves in a line & $S L-W$ & Stamped lines, waves \\
\hline$I H$ & Incised herringbone & $S Q$ & Stamped quadrangles \\
\hline IILS & Incised and infilled lines & $S Q-U$ & $\begin{array}{l}\text { Stamped quadrangles, } \\
\text { unevenly serrated }\end{array}$ \\
\hline$I L S$ & Incised lines & $S S$ & Stylus stabs \\
\hline$I L S B$ & Banded incised lines & SSH & $\begin{array}{l}\text { Spatula stamped } \\
\text { herringbone }\end{array}$ \\
\hline$I L S B-I L S$ & $\begin{array}{l}\text { Banded incised lines, } \\
\text { infilled with incised lines }\end{array}$ & $S S L$ & Spatula stamped line(s) \\
\hline ILSC & Incised lines curvature & $S S L-C$ & $\begin{array}{l}\text { Spatula stamped line(s), } \\
\text { curved }\end{array}$ \\
\hline$I Q$ & Incised quadrangles & SSTB & Spatula stamped triangles \\
\hline$N$ & Nail impressions & $S T-D$ & Stamped triangle(s), dotted \\
\hline$N-S L S$ & $\begin{array}{l}\text { Nail impressions with } \\
\text { stylus-incised lines leading } \\
\text { off them }\end{array}$ & $T D$ & Tear drops \\
\hline$P$ & Seed pits & $T D-C$ & $\begin{array}{l}\text { Tear drops with lines } \\
\text { connecting them }\end{array}$ \\
\hline
\end{tabular}




\begin{tabular}{|l|l|l|l|}
\hline$P S$ & Punctuates, stylus & $U$ & Unknown or worn off \\
\hline$R-C$ & $\begin{array}{l}\text { Roulette, cord } \\
\text { (indeterminate) }\end{array}$ & $W C$ & Wads of clay \\
\hline
\end{tabular}

Pottery motor action codes

Clear distinctions emerge between the types and ratio of décor present in each assemblage. The variability in the décor is greater than seen in the curated assemblages (Brass, 2016; Brass et al., 2018c; Brass and Schwenniger, 2013). The combs used were 2 and 4-toothed.

In Stratum D, the unique décor are flipped cord, fingertip impressions, dragged comb lines, indeterminate cord roulette, stamped dots, angular banded stamped lines, dotted stamped lines (two-toothed comb, not APS), square-toothed comb stamped lines and tear-drops with interconnecting lines.

Stratum C has most of the APS types, excluding dotted paired lines present in the other two assemblages. Other unique décor are impressed widely wrapped cord, comb-dragged channels infilled with stylus-incised lines, joined comb-dragged chevrons, dragged comb V-shaped lines (herringbone), rolled fished spine, impressed hollow reed, banded incised angular lines at opposing angles, overlapping chevrons forming cross-hatching, joined incised chevrons, banded incised lines infilled with incised lines, nail impressions with stylus-incised lines leading off them, stylus punctuates, unevenly serrated comb-stamped channels, and curved spatula-stamped lines.

The combined occurrences for the remaining strata (A and B) are applied clay (unknown motif), impressed cord-wrapped element, lines of rolled cord-wrapped element, fillets, incised dashed lines, incised grooves in a line, plain incised and infilled lines, banded incised lines, incised quadrangles, spatula stamped lines, square-toothed comb stamped lines, square and dotted stamps in the same line, stamped dashed cross-hatching, stamped infilled banded dotted lines, stamped quadrangles, unevenly serrated quadrangles, wads of clay and waves of stamped lines. There are also bands of square-toothed comb stamped lines, stamped dotted lines, stamped dashed lines, dotted droplets and unevenly serrated stamped dots.

\section{The Jebel Moya pottery assemblage: An insight into a social technology}

The provenance of the vast majority of the excavated sherds' clay matrix is local to Jebel Moya. Each phase has its own particularities in the range of tools and motor actions employed. There is now a wider range of Assemblage 1 motifs, which includes the first occurrence of APS dotted paired lines, flipped cord, dragged comb angular lines, incised fan, indeterminate cord roulette, twisted cord roulette, tear drops with connecting lines and banded décor (previously only recognised in Assemblage 3) of stamped lines banded by dragged comb lines. Only the Assemblage 1 sherds from Spit 22 (Trench 2) are burnished, while none are slipped. The 
majority Assemblage 2 sherds are also slipped and burnished except for instances in Trench 6 and the lowermost Phase 2 spits (18 and 19) in Trench 2.

All the Assemblage 3 sherds are slipped and burnished. Assemblage 3 sees the stylus as the dominant tool used in body décor (Table 2), but its décor is a continuation of what is known from the BM collection. Assemblage 2 has the greatest variety of APS, stylus and comb motor actions on the body sherds (Table 9), and also sees the first known instance of rolled fish spine. The one instance of the APS smocking technique from Spit 1 Trench 8 is the first time it is identified at Jebel Moya (see Brass et al., 2018c for an analysis over its appearance at Shaqadud Midden). However, its currently single instance at Jebel Moya is from a mixed context located within the boundaries of where Wellcome's vast camp was situated. All materials from the first two spits of Trench 8 were mixed.

Analyses conducted thus far therefore present the first coherent typology for Jebel Moya pottery. It is acknowledged that this will be refined as excavations progress, particularly in terms of understanding the shape of vessels. Arguably, every classificatory approach is based on attributes, and as Phillips (Phillips 1971) notes, attributes represent one way of describing an artefact. By focusing on the repetition of statistically significant attributes, it is possible to identify the following.

The range of décor on Assemblage 3 rims is fairly narrow in Stratum A but much wider in Stratum B. Assemblage 2 has a wide variety of rim shapes but not so much in décor and similarly Assemblage 1 currently has fewer decorated rims. The latter picture is likely to change as excavation progresses. Overall, there is a much stronger focus on body décor than rim décor, and the motifs and motor actions in Assemblage 2 are particularly diverse.

In terms of shape, Assemblage 3 has the largest variation to date. A comparison with material curated in the BM reveals that Assemblage 3 has a variety of open bowls and jars that tend to be much more bulbous in shape. There are also more open bowls with small handles. Where present, bases are flat and décor starts right above the base. Assemblage 3 also has a number of vessels suitable for storage, including vessels with a straight upper body ideal for the storage of dry goods. Furthermore, there are a number of wide flat platters, of the type that can be used for food sharing, and vessels with a wide opening at the neck.

It is difficult to uncover the whole chaîne opératoire and list of attributes. Some attributes, for example inclusions and their size, may have been items of knowledge known only to potters. What is visible to the naked eye constitutes a public attribute - things that can be seen and experienced by pottery users. Jebel Moya presents a robust sustaining of a technological tradition. This is particularly seen in Assemblage 3's 1200-year time span. It is acknowledged that more radiometric dates could narrow this range somewhat, but it is worth noting that long histories are not an uncommon feature in this part of the world. The modalities in transforming raw materials and the resulting set of operations persist for a long time in Jebel Moya. Such persistence is a strong marker of social groups in which the learning process emphasizes the reproduction of a society's way of doing things. As Lave and Wenger (1991) note, this is how learners construct their social identity during the learning process. 
Ethnoarchaeological studies show that pottery production forms part of a technical specialization that is exclusive to a subgroup of individuals. As Gallay (2007) observes, different ethnic groups within the interior Niger Delta (Mali) have their own ceramic traditions which may appear similar in terms of décor, but which differ in the way vessels are shaped. Indeed, differences in shaping techniques are what differentiate pots made by different socio-cultural groups (Gallay $\&$ Burri-Wyser 2014). Similarly, long-lived traditions are also found in other communities, e.g. the Oromo in Ethiopia (Wayessa 2011). It is not surprising to see this longevity at Jebel Moya, particularly when one takes into account the site's longevity. The present study has highlighted the need for further OSL dates and has informed the strategy for next season's excavation and sampling to elucidate these questions further.

\section{Extending the chronology of Jebel Moya}

Excavations have confirmed Caneva's (1991) contention that there was a late $6^{\text {th }}$ millennium BC occupation of Jebel Moya; currently, it is the earliest known occupation of the site. It was during the mid- $6^{\text {th }}$ millennium BC that the swamps receded from the southern Gezira Plain (Williams and Adamson, 1982), arguably rendering it habitable for the first time during the Holocene. Considering that we have not yet reached bedroom in Trench 2, which continues to yield pottery, there is a strong possibility that more excavations will contribute more comparative data to this ongoing debate.

Currently, the oldest AMS date of 2866-2579 BC (4120 +/1 30 bp, Beta-501556) is on a Ziziphus sp. endocarp from Spit 14 of Trench 2. While the pottery from spits 20 onwards in Trench 2 are Assemblage 1, the sherds from spits 18 and 19 look instead to be an early variant of Assemblage 2: they have stylus-incised angular lines leading directly off each individual fingernail impressions, rolled fish spine and impressed widely-wrapped cord. This, together with the burial from Trench 3 dating to ca. 2350 BC (Brass et al., 2019), raises further questions and requires a reconsideration of the burials unearthed by Wellcome. Only 77 of Wellcome's 3135 human burials had instances of pottery recorded as part of the grave goods (Brass, 2016). Of these 77 burials, 24 contain sherds illustrated on the excavation cards or in Addison's 1949 publication, or both. Of the 24 sherds, 23 are attributable to Assemblage 3 and one to Assemblage 2; the latter is under a hand. The remaining sherds are unidentifiable due to the lack of illustrations, photographs or descriptions.

Relative dating remains extremely useful in so far as analyses are focused and unencumbered by tenuous links to Egyptian archaeology. Historically, Arkell and Addison clashed over dating. Addison (1949) described the site as Napatan (ca. early $9^{\text {th }}$ century -350 BC) in date, whereas, Arkell (Arkell, 1955) attributed a Napatan date to burials 263, 304, 321, 524, 535, 1009 and 1577, and possibly 247 and 1427. He assigned burials 2000b, 2088, 2183, 2193, 2221 and 2225 to the Meroitic on the basis of pottery and associated grave goods. He ascribed painted pottery, with animal (including giraffe), bird and tree motifs, as being inspired by Meroitic painted ware. He claimed that there was a symbol on a pottery fragment (Addison 1949: Plate XCIX, no. 5) which resembled the Meroitic ankh, and that Addison's bowl type G12 was found in a Late Meroitic context at Meroe. It is worth noting that this debate was influenced both by EgyptianNubian studies and a continued desire to attribute a major Nubian or Meroitic discovery to Wellcome (see Vella Gregory 2020). 
Eventually, Addison (1956) abandoned his previous designation of the site as Napatan and assigned it to the Meroitic. The cornerstone of Addison's new argument was burial 1577 (Square J.9, K.10) and burial 2000b from Square I.9, J.10 in the North-East sector of the valley. Burial 1577 (Square J.9, K.10) is recorded $10 \mathrm{~cm}$ below the modern ground surface at the time of Wellcome's expedition and in Stratum B ca. $90 \mathrm{~cm}$ above the surface of Stratum C. A scaraboid was around the neck, which Arkell assigned to the early Napatan (25 ${ }^{\text {th }}$ Dynasty) (Addison, 1949b: Figure 64). It is a Menkare ('Stable is the ka of Ra') scaraboid and not Menkhepera (Piye) as attributed by Addison (1949: 177). This resulted in debates on what is 'proper' Egyptian, ignoring the complex biographies of such objects. Indeed, scaraboids bearing this name have frequently been associated with a 25th Dynasty (Shabaqo) date, but may have a wider chronological appearance (Masson, 2015: 22-23, 29). Of Sudanese manufacture, they can date to 700-500 BC, but their widespread apotropaic usage is likely to have extended their life-cycle beyond the date of manufacture.

Burial $2000 \mathrm{~b}$ was $175 \mathrm{~cm}$ below the modern ground surface, with the base of the grave $35 \mathrm{~cm}$ below the surface of Stratum C. The order of burial within the grave was first individual C, then A and lastly B. A decorated pot (Addison, 1949: Plate CXI, 3 and 4) was in front of B's face at a level of $15-20 \mathrm{~cm}$ above the surface of Stratum C, in Stratum B. This places the pot in the time of early Assemblage 3 as well as in pre-Meroitic times according to the radiometric chronology derived from trenches 1 and 2 (Brass et al., 2019: Table 4). The pot indeed appears to be typically Meroitic in style but the problem is that the chronological sequence for the Napatan period in the central Sudan is missing. The occupation of Meroe stretches back into the Napatan (Humphris et al., 2018) but no pottery chronology is known for the central Sudan from this time, apart from the Meroe Royal Baths assemblage where there is no parallel (Ulrike Nowotnick per. comm. 2019). Furthermore, it is unknown if there had been erosion of the then ground surface at or before the time of burial. In other words, there could have been an erosion channel from rains which was then exploited by being dug in to, resulting in the appearance of $2000 \mathrm{~b}$ being buried before 1577. It means that a reconstruction of burial chronology cannot rest on the sequence of $2000 \mathrm{~b}$ and 1577, and the appearance of a seemingly Meroitic pot in a burial in lower Stratum B cannot have a bearing on the chronological sequence of pottery at Jebel Moya.

None of these rules out there having been an occupation around 2000 years ago. There was a large pot embedded in a calcium carbonate feature in Stratum A of Square M.5, N.6 (Addison, 1949b: Plate CXI). The décor strongly suggests that Arkell's (1955) attribution to the Meroitic is accurate. Other objects found in burials are also considered. These include a light green glazed scarab in burial 2225a, (Addison, 1949a: Plate XLV, B4; Addison, 1949b), a bronze statuette of the Egyptian god Shu found c. $15 \mathrm{~cm}$ from the head of burial 524 and a pale green faience Udat amulet with burial 535. There are more recorded instances in non-burial contexts: Table 3.12 in Brass (2016) lists the occurrences of surface finds of plaques and scarabs in multiple areas across the site, and their chronological attribution of manufacture by Addison (1949a: 117). The ages of manufacture, not deposition, range around the mid- $1^{\text {st }}$ millennium to the $4^{\text {th }}$ century BC.

There are also occurrences which have no recorded provenances. The wedjat eyes in the first row of amulets in Plate XLIX are so far from a recognisable Egyptian form that they could arguably 
be from the Meroitic era. In Plate L, scarab number 8 has the name of the Egyptian Pharoah Sheshonq I (ca. 960 BC) but the rest of the scarabs in the same plate very different from examples found in Egypt. The cartouches are also not authentically Egyptian, but Geoffrey Tassie (pers. comm. 2019) suggested that the hippo and fly amulets would seem to indicate an end of the $2^{\text {nd }}$ millennium BC date of manufacture. It is worth noting that at such distances, people are likely to have valued objects that looked Egyptian without requiring any 'authenticity'. Such objects would acquire their own meanings (for a similar phenomenon see Vella 2010).

Dating a site with a long history of occupation remains an ongoing process. A review of curated and excavated material shows occupation from the $2^{\text {nd }}$ millennium $\mathrm{BC}$ down into the early first millennium $\mathrm{AD}$, after which activity ceased. It is also becoming clear that there were intensive earlier occupations currently stretching back to the late sixth millennium BC. Burial activity had started by 2400 BC (Brass et al., 2019), the intensity of which is currently unknown, and certainly continued in the final occupation phase (Brass, 2016).

\section{Implications and future directions}

Analyses point to a longevity in technological traditions and a variability of tools and décor within assemblages. The latter is a common feature of a number of sites, even ones $250 \mathrm{~km}$ distant (see for example work at Kadero and el Geili by Caneva (1988) and Chłodnicki et al. (2011)). These have often been used to make tenuous connections between distant sites and ways of life, but this merely shows the importance of variability to different groups of people. A further problem is the use of Eurocentric terminology, resulting in the use of the term Neolithic to merely refer to the presence of domesticated plants and animals. The Neolithic in the central Sudan is currently dated from the $5^{\text {th }}$ to the $3^{\text {rd }}$ millennium BC (Edwards, 2004; Sadig, 2013, 2012). Even within European and Mediterranean archaeology there is no one type of Neolithic and the Sudan is no exception. Excavations at Al Khiday (immediately south of Omdurman) and Shaqadud (north-western boundary between the Nile and the Butana Plain) on the margins of the Nilotic Central Sudan show dissimilarities within their Neolithic pottery assemblages compared with their Nilotic counterparts (Brass et al., 2018c; Caneva and Marks, 1990; Dal Sasso et al., 2014; Mohammed-Ali, 1991; Salvatori, 2012; Usai and Salvatori, 2019). Indeed, Shaqadud's Neolithic assemblages have been interpreted as savannah rather than Nilotic-oriented and this distinction is a feature of the Sudanese Neolithic.

The start of the Early Neolithic at Kadero is dated to ca. 4560 BC and the Late Neolithic ca. 3830 BC (Chłodnicki et al., 2011). In general, the pottery technology remained broadly similar, while décor, vessel forms and the relative preponderance of select tools differed. El Geili is another prominent site with a substantial Neolithic component, but contextualising its Neolithic pottery is difficult due to reworking, development and deflation (Caneva, 1988). El Geili has a single date from a freshwater mollusc for the Neolithic, 4683 - $4236 \mathrm{BC}(5570+/-100 \mathrm{bp}$, T5022). A study of décor and motor actions should be standard across sites. This is not because décor provides an absolute date, but because as we have shown, it is a useful means to answer broader questions while providing methodological clarity. At Jebel Moya, it is noted that some of 
Assemblage 3 could broadly fall within the 'Neolithic', as could some of Assemblage 2. It is clear that site occupation predates radiometric dates. The following points are noted.

Sherds from Trenches 6 and 8 bear strong similarities to others found at Kadero and other sites. The rough stylus-incised chevrons on a rim from Spit 3 in Trench 6 are similar to ones from Kadero (Chłodnicki et al., 2011: Figure 6.A3). In both spits 3 and 4, there are stylus-incised cross-hatchings and stylus-incised angular lines on rims similar to Shaheinab (Arkell, 1953: Plates 37.14 and 37.22) and Kadero (Chłodnicki et al., 2011: Figures 6.A2 and 6.A4). In Spit 5 (Figure 6a), a sherd has evenly serrated comb rocker-stamped, packed zigzag dotted lines across both the body and rim. It is similar to Figure 12.6 at Kadero and Figure 8.6 at el Geili. In Spit 8, the occurrences of alternating pivoting stamp (APS) are similar to el Geili (Caneva, 1988: Figure 12.5). Similar APS lines are also present later on at Shaheinab (Arkell, 1953: Plate 41.12), this time covering part of the whole vessel and labelled "Protodynastic", a designation meaning it was at the end or shortly after the Late Neolithic. There are further instances of similarities with sherds from el Geili: APS (Figure 6b) (Caneva, 1988: Figure 13.7), cross-hatching (Figure 6c) and vertical comb-incised lines.

From Trench 8's Spit 1, a straight-rimmed sherd (Figure 7a) has packed, incised chevrons crisscrossing to also form cross-hatching on the body immediately below the rim. Kadero has similar (Chłodnicki et al., 2011: Fig. 6.A2). Also from Spit 1, Figure 7b is the same as Figure 12.5 from el Geili and Subtype LA2 at Kadero: A series of two APS dotted lines covering the surface of burnished, fine ware sherds which are attributed to the Early Neolithic at Kadero. In Spit 2, the APS double-pronged wavy line has counterparts at el Geili (Caneva, 1988: Fig. 12.2b). A further two examples from Trench 2, assigned to early Assemblage 2 (Spit 19; Figure 8a) and Assemblage 1 (Spit 22; Figure 8b), share the same potter's stamp. It is present in northern Sudan at Djabarona 84/13, dated to 3000 - 2500 BC (Keding, 1997: Fig. 3.8). Similar sherds are widespread over the Sahara, e.g. in the Taoudenni Basin's (Mali) assemblages from the midHolocene (Commelin, 1983). However, the stamp is not diagnostic of a particular phase or time period.

Haaland (1987) has noted the her site of Rabak has the same thick, everted and rolled rims found in Jebel Moya's Assemblage 2. The Rabak samples were from Layer 2 which is dated to 3378 2909 BC (4490 +/- 100 bp, T-5132). There are question marks over the reliability of the date due to uncertainty of the relationship between the shell sample and the pottery, and the potential freshwater reservoir effect (FRE) which may have yielded too early a date due to 'hard water' with dissolved old carbonates. The same thick, everted and rolled rim sherds are also found in the vicinity of Aba Island slightly the north of Rabak by one of the authors (Vella Gregory) and Ahmed Adam (co-director of the project) during a surface survey.

At Al Khiday, petrographic analysis of Mesolithic and Neolithic sherds from ca. $7000-4000$ BC show three main groups: alkali-feldspar inclusions (Group 1, Mesolithic) and quartz inclusions (groups 2 and 3). Groups 2 (Mesolithic) and 3 (Neolithic) were differentiated based on the size, number and distribution of the inclusions (Dal Sasso et al., 2014). While the quartz inclusions were obtained from either riverine or aeolian deposits, they were added to clay containing plagioclase, alkali feldspar, biotite, chlorite, white mica, amphibole, clinopyroxene and opaque minerals which was not obtained from White Nile or main Nilotic deposits. Similar pottery 
pastes are known from elsewhere in the central Sudan (see, for example, Chłodnicki, 1989; Khabir, 1991; Klein et al., 2004). For Group 1, the alkaline granite may have originated from Sabaloka or Jebel Seleitat, before the $6^{\text {th }}$ Cataract north of Khartoum. Transport was likely by riverine boats (Peters, 1991; Van Neer, 1989), with an early Mesolithic boat motif found at Al Khiday (Usai and Salvatori, 2007). The presence of riverine transport would have facilitated connectivity between the southern Gezira and the central Sudan.

At present, these similarities raise more questions and provide exciting future directions. Overall, it is clear that the relationship between the southern Gezira and central Sudan is much earlier than currently recognized. These similarities need to result in a broader investigation of contact. This study highlights the need for a more systematic and comprehensive radiometric dating programme across the central and south-central Sudan, one rooted in careful excavation. Results from Kadero, showing potentially earlier contact, indicate that this could significantly change the current state of knowledge. Finally, Jebel Moya illustrates that a widespread focus on the Meroitic state has vastly over-hypothesized its spread. The Meroitic state (ca. 350 BC) is distinguished by standardized pottery production. Its absence at Jebel Moya shows that contra Addison and Arkell, Meroitic rule did not extend that far south (Brass, 2016; Brass et al., 2019).

\section{Conclusion}

Curated and excavated pottery from Jebel Moya has been analyzed via attribute analysis, resulting in the first secure reconstruction of pottery sequencing. The results extend the known range of tools and motor actions employed, extend the chronology and provide a solid framework for continued studies. A theoretically informed method is enabling inroads into the study of technology in Sudanese archaeology, highlighting numerous issues along the way. Archaeological and anthropological data show that relying on a superficial similarity in décor is unhelpful both in terms of chronologies and in understanding the communities under study.

The importance of the persistence of motifs has long been established in archaeology and anthropology (see for example Deetz 1965). It is only by methodically tracing the occurrence of décor that we can identify persistence and its meaning. This study acknowledges that ceramic production is one facet of community of practice (sensu Lave \& Wenger 1991:8), which also includes the multi-directional transfer of knowledge). Our approach has considered a unit of analysis that includes histories of learning (Stahl 2016), which in this case persisted over a long period of time.

Questions remain on the persistence of burial activity. It started from at least the $3^{\text {rd }}$ millennium $\mathrm{BC}$ and further investigation will clarify whether this was continuous or in distinct phases. New knowledge of the stratigraphy, pottery sequencing and the radiometric dates gives a clearer chronological sequence for burials. The chronological continuities or discontinuities between the late $6^{\text {th }}$ millennium $\mathrm{BC}$ and the subsequent occupations are clearly in need of further investigation that includes a comprehensive radiometric dating programme. Similarly, assemblages from other sites show the need for more targeted investigations across the central Sudan, particularly to delineate population movement and networks. There are strong indications that these go back further than the $3^{\text {rd }}$ millennium BC. It remains to be determined whether life in the late $6^{\text {th }}$ millennium $\mathrm{BC}$ was more mobile or, as noted at Al Khiday (Usai and Salvatori, 
654 2019), a form of organized sedentism. It is clear that agro-pastoralism was practised at least as 655 early as the $3^{\text {rd }}$ millennium BC. Continued research will focus on refining the timings and 656 complexity and how the nature of occupation at Jebel Moya compares with central Sudan 657 (Salvatori \& Usai, 2019; Brass, 2016).

658

659 Disentangling the biography of Jebel Moya therefore remains an ongoing process. The scholarly 660 biography of Jebel Moya has been particularly fragmented, resulting in a number of approaches 661 that do not take into account people and knowledge (Addison, 1949b; Gerharz, 1994). Studies 662 need to be foregrounded in everyday practice and how they materialize world-views (sensu 663 Bourdieu 1977). As such, a study of technology is also one of cultural choices. Ongoing and 664 future research at Jebel Moya will continue to focus on integrating data sets that have been 665 treated as separate (tools, botanical remains, human remains etc) to arrive at a more nuanced 666 reading of this site.

667

668

669

670

671

672

673

674

675

676

677 


\section{Acknowledgements}

679 We are grateful to the people of Jebel Moya for their generous enthusiasm, support and

680 hospitality, and to our fantastic fieldwork team. We are also grateful to the National

681 Corporation of Antiquities and Museums (NCAM) and the Society for Libyan Studies for

682 their continued support. We thank the anonymous reviewers for the valuable feedback. This

683 paper is dedicated to the memory of Alessandro Salvatori, who will always be fondly

684 remembered for his generosity of spirit and his enormous contribution to the Sudan.

685

686 Funding

687 The excavation of Jebel Moya is funded by The Society for Libyan Studies. 


\section{References}

Adams, W., Adams, E., 1991. Archaeological typology and practical reality: A dialectical approach to artifact classification and sorting. Cambridge University Press, Cambridge.

Addison, F., 1949a. Wellcome Excavations in the Sudan: II, Jebel Moya, 1910-1914. Oxford University Press, Oxford.

Addison, F., 1949b. Wellcome Excavations in the Sudan: I, Jebel Moya, 1910-1914. Oxford University Press, Oxford.

Arkell, A.J, 1949. Early Khartoum. Oxford University Press, Oxford.

Arkell, A.J., 1955. Jebel Moya, by Addison Frank, with a chapter by Lacaille A. D.. Vols. 1 (text) and II (plates) of the Wellcome Excavations in the Sudan, pp. XIV + 399, 123 text figs., CXVI pls. Oxford University Press, 1949. £6 6s. Proc. Prehist. Soc. 20, 126-130. https://doi.org/10.1017/S0079497X00017886

Arkell, A.J., 1953. Shaheinab: An account of the excavation of a Neolithic occupation site carried out for the Sudan Antiquities Service in 1949-50. Oxford University Press, Oxford.

Bourdieu, P. 1977. Outline of a Theory of Practice. Cambridge: Cambridge University Press.

Brass, M., 2016. Reinterpreting chronology and society at the mortuary complex of Jebel Moya (Sudan). Cambridge Monographs in African Archaeology 92, Archaeopress, Oxford.

Brass, M., Adam, A., Fuller, D.Q., Stevens, C., Hassan, F., Kozieradzka-Ogunmakin, I., Abdallah, R., Alawad, O., Abdalla, A., Wellings, J., Abdelrahman, A., 2018a. First season of the UCL - UoK - NCAM Expedition 38 to the Southern Gezira (Sudan): Jebel Moya. Sudan \& Nubia 22, 38-45.

Brass, M., Adam, A., Fuller, D.Q., Stevens, C., Hassan, F., Kozieradzka-Ogunmakin, I., Abdallah, R., Alawad, O., Abdalla, A., Wellings, J., Abdelrahman, A., 2018b. Jebel Moya: new excavations at the largest pastoral burial cemetery in sub-Saharan Africa. Antiquity 92, e6. https://doi.org/10.15184/aqy.2018.226

Brass, M., Adam, A., Vella Gregory, I., Abdallah, R., Alawad, O., Abdalla, A., KozieradzkaOgunmakin, I., Wellings, J., Albadwi, A., Le Moyne, C., Hassan, F., Abdelrahman, A., 2020. The second season of excavations at Jebel Moya (south-central Sudan). Libyan Stud. 51, In Press.

Brass, M., Adam, A., Wellings, J., 2018c. New data from Jebel Moya and Shaqadud (central Sudan): implications for Late Mesolithic interconnectivity with the Sahara. Libyan Stud. 49, 21-49. https://doi.org/10.1017/lis.2018.16

Brass, M., Fuller, D.Q., Macdonald, K., Stevens, C., Adam, A., Kozieradzka-Ogunmakin, I., Abdallah, R., Alawad, O., Abdalla, A., Vella Gregory, I., Wellings, J., Hassan, F., Abdelrahman, A., 2019. New findings on the significance of Jebel Moya in the eastern Sahel. Azania Archaeol. Res. Africa 54, 425-444.

Brass, M., Schwenniger, J.-L., 2013. Jebel Moya (Sudan): New dates from a mortuary 
complex at the southern Meroitic frontier. Azania 48, 455-472. https://doi.org/10.1080/0067270X.2013.843258

Camps-Fabrer, H., 1966. Sur quelques techniques décoratives de la céramique impressionnée saharienne, in: scientifique, S. national de la recherche (Ed.), Congrès Préhistorique de France: Compte Rendu de La XVIIIe Session. Société Préhistorique Francaise, Paris, pp. $143-154$.

Caneva, I., 1991. Jebel Moya revisited: A settlement of the 5th millennium BC in the middle Nile basin. Antiquity 65, 262-268.

Caneva, I., 1988. El Geili: The history of a Middle Nile environment 7000 BC - AD 1500. BAR International Series 424, Cambridge Monographs in African Archaeology 29. Oxford.

Caneva, I., 1987. Pottery decoration in prehistoric Sahara and Upper Nile: a new perspective, in: Barich, B. (Ed.), Archaeology and Environment in the Libyan Sahara: The Excavations in the Tadrart Acacus, 1978-1983. BAR International Series, 368, Oxford, pp. 231-254.

Caneva, I., Marks, A., 1990. More on the Shaqadud Pottery: Evidence for Saharo-Nilotic Connections during the 6th-4th Millennium B.C. Archéologie du Nil Moyen 4, 11-35.

Chłodnicki, M., 1989. The petrographic analyses of the Neolithic pottery of Central Sudan, in: Krzyźaniak, L., Kobusiewicz, M. (Eds.), Late Prehistory of the Nile Basin and the Sahara. Poznan Archaeological Museum, Poznan, pp. 369-373.

Chłodnicki, M., Kobusiewicz, M., Kroeper, K., 2011. Kadero: The Lech Krzyźaniak excavations in the Sudan.

Commelin, D., 1983. Ceramique, in: Sahara Ou Sahel? Quaternaire Recent Du Bassin de Taoudenni (Mali). CNRS, Paris, pp. 343-366.

Dal Sasso, G., Maritan, L., Salvatori, S., Mazzoli, C., Artioli, G., 2014. Discriminating pottery production by image analysis : a case study of Mesolithic and Neolithic pottery from Al Khiday ( Khartoum, Sudan ). J. Archaeol. Sci. 46, 125-143. https://doi.org/10.1016/j.jas.2014.03.004

Deetz, J. 1965. The Dynamics of Stylistic Change in Arikara Ceramics. Tucson: University of Arizona Press.

Edwards, D., 2004. The Nubian past: an archaeology of the Sudan. Routledge, London.

el Mahi, A., Haaland, R., 1984. Archaeological research in the area of Rabak and Atbara, Sudan. Nyame Akuma 24/25, 28-32.

Gatto, M., 2011a. The Nubian pastoral culture as link between Egypt and Africa: A view from the archaeological record, in: Exell, K. (Ed.), Egypt in Its African Context: Proceedings of the Conference Held at The Manchester Museum,University of Manchester, 2-4 October 2009 . BAR International Series 2204, Oxford, pp. 21-29.

Gatto, M., 2011b. The relative chronology of Nubia. Archéo-Nil 21, 81-100.

Gatto, M., 2006. The Khartoum Variant pottery in context: Rethinking the Early and Middle Holocene cultural sequence. Archéologie du Nil Moyen 10, 57-72.

Gerharz, R., 1994. Jebel Moya. Akademie Verlag. Berlin, (Meroitica 14). Berlin. 
Haaland, R., 1987. Socio-economic differentiation in the Neolithic Sudan. B.A.R, Oxford.

Haaland, R., 1984. Continuity and discontinuity: How to account for a two thousand years gap in the cultural history of the khartoum nile environment. Nor. Archaeol. Rev. 17, 39-51. https://doi.org/10.1080/00293652.1984.9965397

Haour, A., Manning, K., Arazi, N., Gosselain, O., Guèye, N., Keita, D., Livingstone Smith, A., MacDonald, K., Mayor, A., McIntosh, S., Vernet, R., 2010. African pottery roulettes past and present: Techniques, identification and distribution.

Hays, T., 1971. The Sudanese Neolithic: A critical analysis. Southern Methodist University, Unpublished $\mathrm{PhD}$ dissertation, Dallas.

Humphris, J., Bussert, R., Alshishani, F., Scheibner, T., 2018. The ancient iron mines of Meroe. Azania Archaeol. Res. Africa 53, 291-311. https://doi.org/10.1080/0067270X.2018.1515922

Jesse, F., 2010. Early pottery in Northern Africa - An overview. J. African Archaeol. 8, 219238.

Jesse, F., 2002. Wavy line ceramics: Evidence from Northeastern Africa, in: Nelson, K., Associates (Eds.), Holocene Settlement of the Egyptian Sahara (Volume 2): The Pottery of Nabta Playa. Kluwer Academic/Plenum Publishers, New York, pp. 79-96.

Keding, B., 1997. Djabarona 84/13. Africa Praehistorica 9, Koeln.

Keech McIntosh, S., 1995. Pottery, in: McIntosh, S. (Ed.), Excavations at Jenne-Jeno, Hambarketolo, and Kaniana (Inland Niger Delta, Mali), the 1981 Season. University of California Press, Berkeley, pp. 130-213.

Khabir, A., 1991. The firing index of Neolithic pottery from Central Nile. Nyame Akuma 35, 33-35.

Klein, M., Jesse, F., Kasper, H., Golden, A., 2004. Chemical characterization of ancient pottery from Sudan by X-ray fluorescence spectrometry (XRF), electron microprobe analysis (EMPA) and inductively coupled plasma mass spectrometry (ICP-MS). Archaeometry 46, 339-356.

Lave, J. \& Wenger, E. 1991. Situated Learning: Legitimate Peripheral Participation. Cambridge: Cambridge University Press.

MacDonald, K., 2011. Betwixt Tichitt and the IND: The pottery of the Faïta Facies, Tichitt Tradition. Azania 46, 49-69.

Marks, A., Mohammed-Ali, A., Peters, J., Robertson, R., 1985. The Prehistory of the Central Nile Valley as seen from the Eastern Hinterlands: Excavations at Shaqadud, Sudan. J. F. Archaeol. 12, 262-272.

Masson, A., 2015. Scarabs, scaraboids and amulets, in: Villing, A., Bergeron, M., Bourogiannis, G., Johnston, A., Leclère, F., Masson, A., Thomas, R. (Eds.), Naukratis: Greeks in Egypt. British Museum, London, pp. 3-96.

Mayor, A., Huysecom, E., Gallay, A., Rasse, M., Ballouche, A., 2005. Population dynamics and Paleoclimate over the past 3000 years in the Dogon Country, Mali. J. Anthropol. Archaeol. 24, 25-61.

Mohammed-Ali, A., 1991. The Mesolithic and Neolithic ceramics from Shaqadud Midden, 
in: Marks, A., Mohammed-Ali, A. (Eds.), The Late Prehistory of the Eastern Sahel: The Mesolithic and Neolithic of Shaqadud, Sudan. Southern Methodist University Press, Dallas, pp. 65-94.

Mohammed-Ali, A., Khabir, A., 2003. The Wavy Line and the Dotted Wavy Line Pottery in the Prehistory of the Central Nile and the Sahara-Sahel Belt. African Archaeol. Rev. 20, $25-58$.

Nordstrom, H., 1972. A qualitative analysis of the Early and Middle Nubian pottery, in: Nordstrom, H. (Ed.), Neolithic and A-Group Sites. Scandanavian University Books, Stockholm, pp. 33-96.

Peters, J., 1991. Mesolithic fishing along the Central Sudanese Nile and Lower Atbara. Sahara 4, 33-40.

Sadig, A., 2013. Reconsidering the 'Mesolithic' and 'Neolithic' in Sudan, in: Shirai, N. (Ed.), Neolithisation of Northeastern Africa. ex oriente, Studies in Early Near Eastern Production, Subsistence, and Environment 16. Berlin, pp. 23-42.

Sadig, A., 2012. Chronology and cultural development of the Sudanese Neolithic. Beiträge zur Sudanforsch. 11, 137-184.

Salvatori, S., 2012. Disclosing archaeological complexity of the Khartoum Mesolithic: New data at the site and regional Level. African Archaeol. Rev. 29, 399-472.

Salvatori, S. \& Usai, D. 2019. The Neolithic and 'Pastoralism' Along the Nile: A dissenting view. Journal of World Prehistory 32: 251-285. https://doi.org/10.1007/s10963-01909132-1

Salvatori, S., Usai, D., 2008. A Neolithic cemetery in the Northern Dongola Reach. Excavations at Site R12.

Salvatori, S., Usai, D., 2007. The Sudanese Neolithic revisited. Crippel 26, 323-333.

Salvatori, S., Usai, D. \& Zerboni, A. Mesolithic Site Formation and Palaeoenvironment Along the White Nile (Central Sudan). Afr Archaeol Rev 28, 177-211 (2011). https://doi.org/10.1007/s10437-011-9095-3

Stahl, A. 2016. Crafting life in turbulent times: Communities of practice in the western Volta Basin from the 13th to the 17th century A.D, in: Roddick, A. \& Stahl, A. (ed.) Knowledge in Motion: Constellations of Learning Across Time and Space. Tucson: University of Arizona Press, pp.179-215.

Usai, D., Salvatori, S., 2019. Azania : Archaeological Research in Africa The Mesolithic period on the White Nile region and the al-Khiday sites The Mesolithic period on the White Nile region and the 5534. https://doi.org/10.1080/0067270X.2019.1691846

Usai, D., Salvatori, S., 2007. The oldest representation of a Nile boat. Antiquity 81, 314.

Van Neer, W., 1989. Fishing along the Nile, in: Krzy_zaniak, L., Kobusiewicz, M. (Eds.), Late Prehistory of the Nile Basin and the Sahara. Poznan Archaeological Museum, Poznan, pp. 49-56.

Vella Gregory, I., 2020. Ordering the land beyond the Sixth Cataract: Imperial policy, archaeology and the role of Henry Wellcome. Libyan Stud. 51: 43-60. 
853

854

855

856

857

858

859

860

861

862

863

864

Vella Gregory, I., 2017. The application of pottery attribute analysis: A case-study from the Neolithic complex of Kordin, Malta. J. Archaeol. Sci. Reports 14, 543-556. https://doi.org/10.1016/j.jasrep.2017.06.032

Vella, N., 2010. "Phoenician" metal bowls: Boundary objects in the Archaic period. Boll. di Archeol. Online I, 21-37.

Williams, M., Adamson, D., 1982. A land between two Niles: Quaternary geology and biology of the Central Sudan.

Winchell, F., 2013. The Butana Group ceramics and their place in the Neolithic and PostNeolithic of Northeast Africa. BAR, Cambridge Monographs in African Archaeology 83, Oxford. 
865

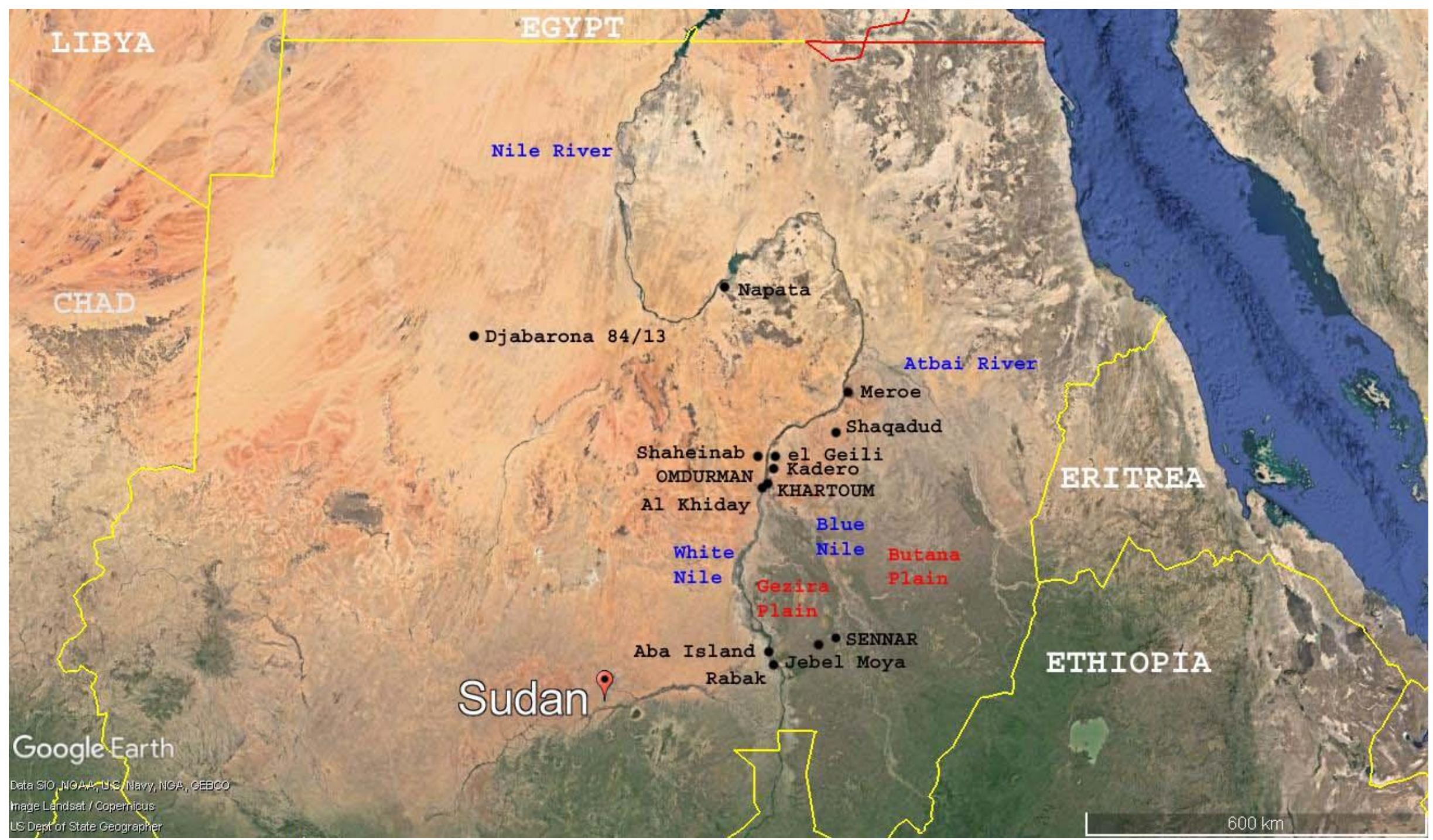

Figure 1. Jebel Moya (Gezira Plain, south-central Sudan) in relation to Khartoum and the other major sites cited. 


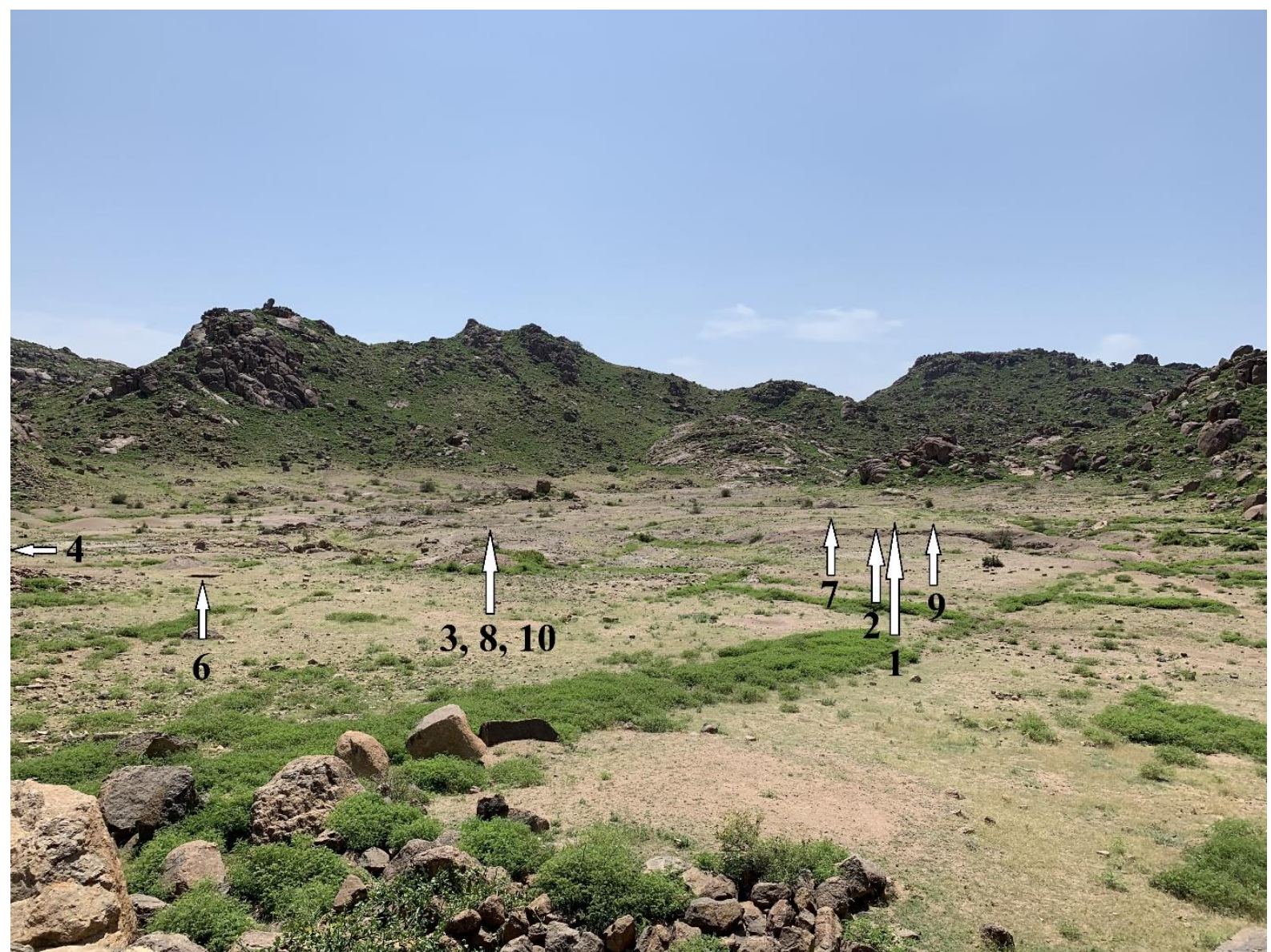

871 Figure 2. Location of the trenches. Photograph taken facing south from the House of the 872 Boulders

873
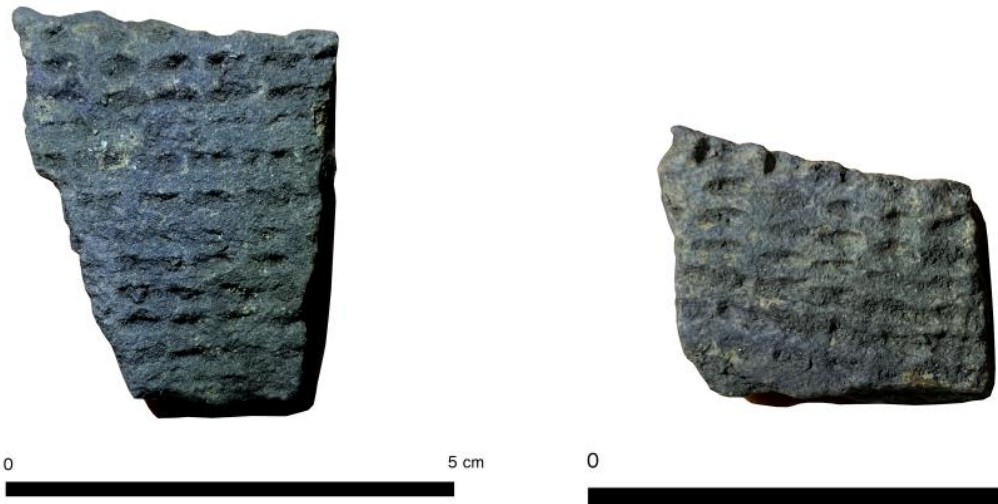

874

875

876

877

Figure 3 (a, b). Assemblage 1 sherds.

878 

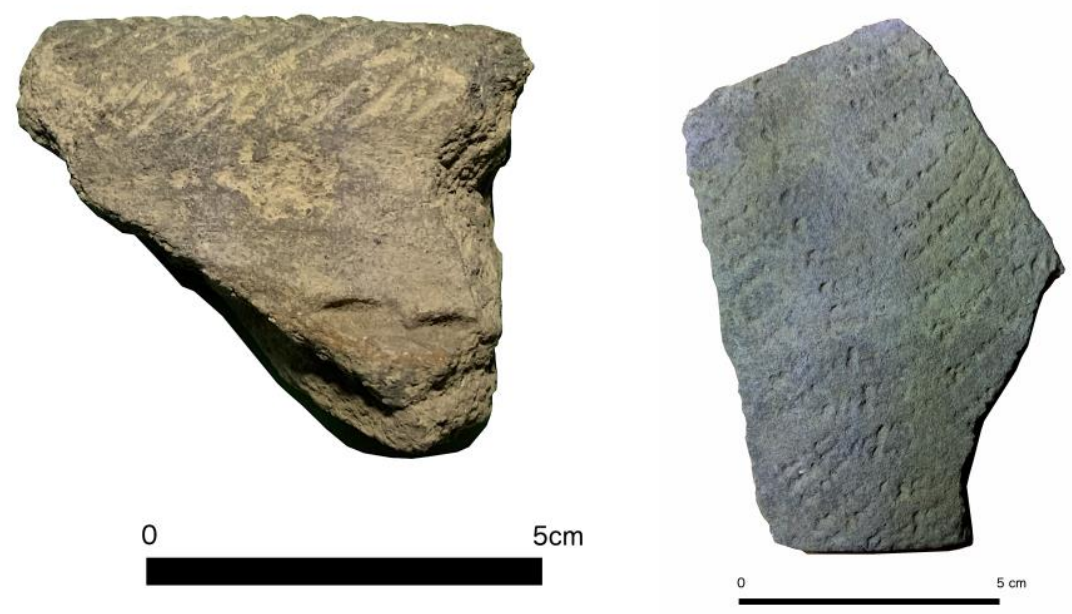

880

881

Figure 4 (a, b). Assemblage 2 sherds.

882

883

Figure 5 (a, b). Assemblage 3 sherds.
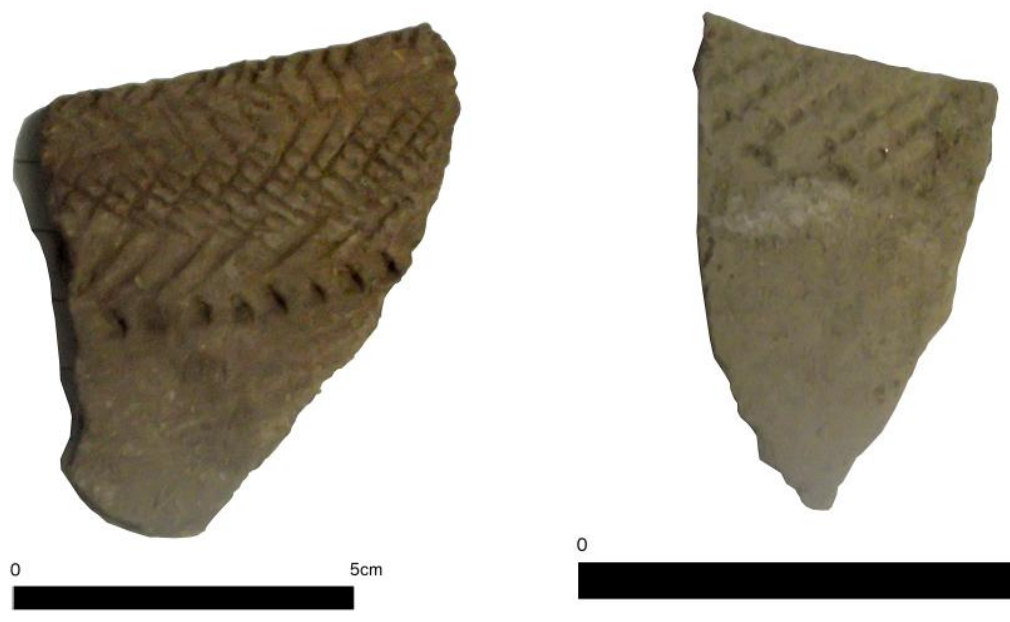

888

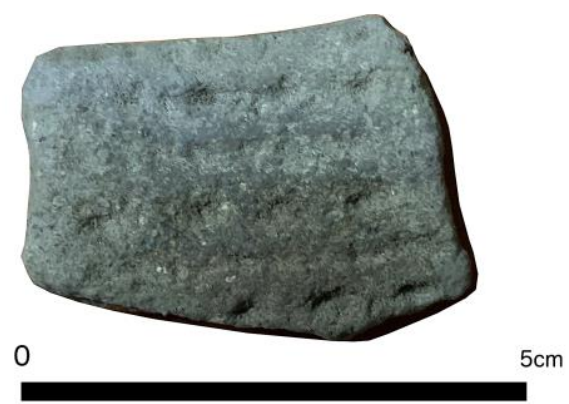


892 Figure 6a. Trench 6, Spit 5. Evenly serrated comb rocker-stamped, packed zigzag dotted lines 893 across both the body and rim.

894
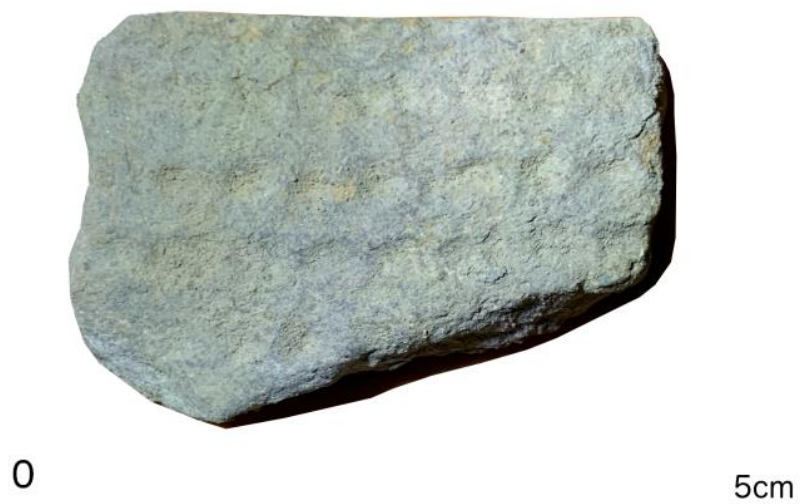

895

896

897

898

899

Figure 6b. Trench 6, Spit 5. APS décor.

Figure 6c. Trench 6, Spit 5. Cross-hatching décor. 


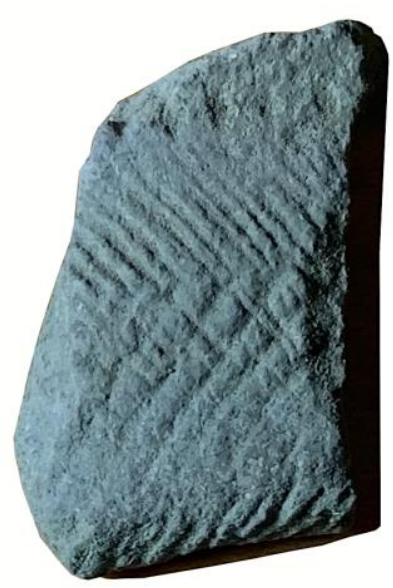

0

$5 \mathrm{~cm}$

904

905

906

907

908

909
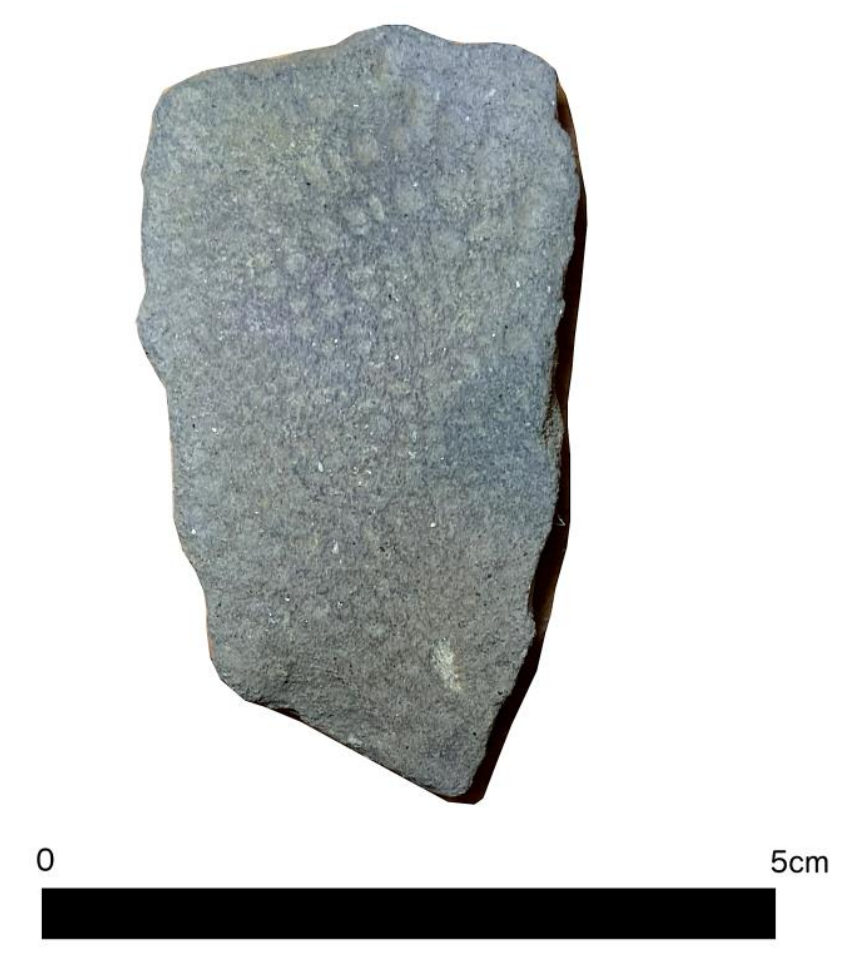
burnished, fine ware sherd.
Figure 7a. Trench 8, Spit 1. A straight-rimmed sherd with packed, incised chevrons crisscrossing to also form cross-hatching on the body immediately below the rim.

Figure 7b. Trench 8, Spit 1. A series of two APS dotted lines covering the surface of a 

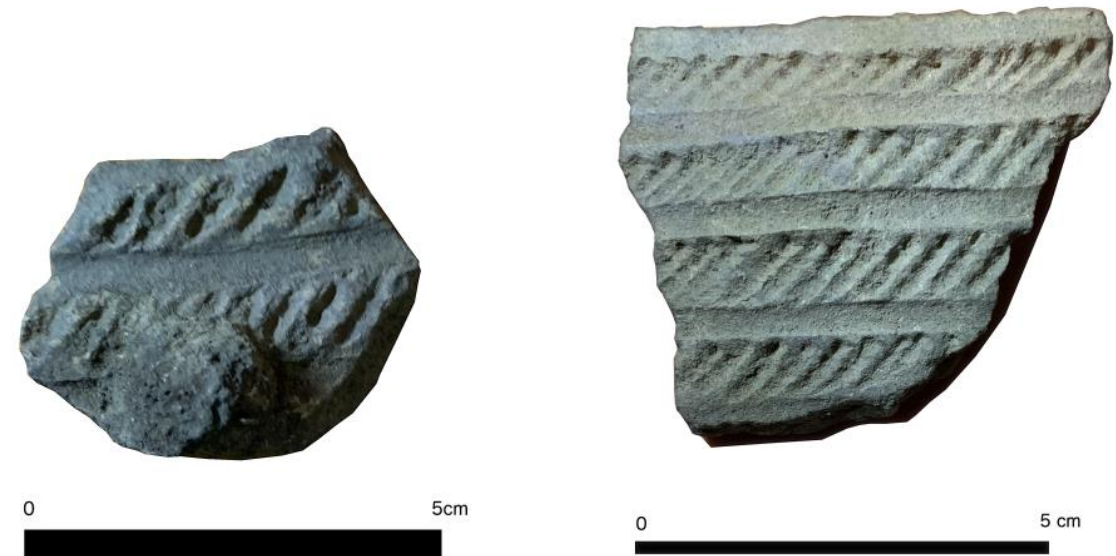

Figures 8a and b. Trench 8, Spit 2. Shared potter's stamp present in north Sudan (Djabarona 84/13) and widespread over the Sahara.

\begin{tabular}{|l|l|l|l|c|}
\hline \multicolumn{1}{|c|}{ Material } & Context & \multicolumn{1}{|c|}{$\begin{array}{c}\text { Lab } \\
\text { number }\end{array}$} & $\begin{array}{c}\text { Age }{ }^{\mathbf{1 4} C} \\
\text { (bp) }\end{array}$ & $\begin{array}{c}\text { Calibrated } \\
\text { age }\end{array}$ \\
\hline Molar dental enamel & Trench 3 & GdA-5760 & $\begin{array}{l}3880 \pm \\
40\end{array}$ & $\begin{array}{c}2470-2210 \\
\text { BC }\end{array}$ \\
\hline Sorghum grain & $\begin{array}{l}\text { Trench 1, } \\
\text { Spit 2 }\end{array}$ & $\begin{array}{l}\text { Beta- } \\
501555\end{array}$ & $\begin{array}{l}3930 \pm \\
30\end{array}$ & $\begin{array}{c}2558-2300 \\
\text { BC }\end{array}$ \\
\hline $\begin{array}{l}\text { Capra/Ovismaxillary } \\
\text { molar }\end{array}$ & $\begin{array}{l}\text { Trench 2, } \\
\text { Spit 5 }\end{array}$ & $\begin{array}{l}\text { OxA-X- } \\
3000-40\end{array}$ & $\begin{array}{l}2473 \pm \\
21\end{array}$ & $\begin{array}{c}766-509 \\
\text { BC }\end{array}$ \\
\hline $\begin{array}{l}\text { Bos maxillary } \\
\text { premolar }\end{array}$ & $\begin{array}{l}\text { Trench 2, } \\
\text { Spit 12 }\end{array}$ & $\begin{array}{l}\text { OxA-X- } \\
3000-39\end{array}$ & $\begin{array}{l}3269 \pm \\
22\end{array}$ & $\begin{array}{c}1613-1502 \\
\text { BC }\end{array}$ \\
\hline Sorghum husks & $\begin{array}{l}\text { Trench 2, } \\
\text { Spit 14 }\end{array}$ & $\begin{array}{l}\text { Beta- } \\
501557\end{array}$ & $\begin{array}{l}3970 \pm \\
30\end{array}$ & $\begin{array}{c}2575-2350 \\
\text { BC }\end{array}$ \\
\hline $\begin{array}{l}\text { Ziziphus sp. } \\
\text { endocarp }\end{array}$ & $\begin{array}{l}\text { Trench 2, } \\
\text { Spit 14 }\end{array}$ & $\begin{array}{l}\text { Beta- } \\
501556\end{array}$ & $\begin{array}{l}4120 \pm \\
30\end{array}$ & $\begin{array}{c}2866-2579 \\
\text { BC }\end{array}$ \\
\hline Sorghum husks & $\begin{array}{l}\text { Trench 4, } \\
\text { Spit 9 }\end{array}$ & $\begin{array}{l}\text { Beta- } \\
501554\end{array}$ & $\begin{array}{l}3870 \pm \\
30\end{array}$ & $\begin{array}{c}2465-2211 \\
\text { BC }\end{array}$ \\
\hline
\end{tabular}

Table 1a. AMS dates on botanical, faunal and human remains from trenches 1,2, 3 and 4 . The 
924 Trench 1 Spit 2 date is regarded as intrusive (Brass et al. 2019). Dating was done by Beta

\begin{tabular}{|c|c|c|c|c|}
\hline $\begin{array}{c}\text { Laboratory } \\
\text { code }\end{array}$ & $\begin{array}{c}\text { Brass' } \\
\text { Assemblage } \\
\text { attribution }\end{array}$ & $\begin{array}{c}\text { Previous OSL } \\
\text { age estimate } \\
\text { (years before } \\
\mathbf{2 0 1 2} \text { ) }\end{array}$ & $\begin{array}{c}\text { Revised OSL } \\
\text { age estimate } \\
\text { (years before } \\
\mathbf{2 0 1 9 )}\end{array}$ & $\begin{array}{c}\text { Revised } \\
\text { calibrated dates }\end{array}$ \\
\hline & 3 & $1760 \pm 295$ & $1880 \pm 300$ & $161 \mathrm{BC}-\mathrm{AD}$ \\
X5291 & 2 & $3245 \pm 755$ & $3510 \pm 795$ & 439 \\
X5292 & 3 & $1490 \pm 270$ & $1620 \pm 295$ & $2286-696 \mathrm{BC}$ \\
X5293 & 2 & $3435 \pm 260$ & $3720 \pm 205$ & AD 104-694 \\
X5294 & 2 & $3250 \pm 445$ & $3480 \pm 435$ & $1906-1496 \mathrm{BC}$ \\
X5295 & 3 & $1545 \pm 535$ & $1680 \pm 575$ & $1896-1026 \mathrm{BC}$ \\
X5296 & & & & $236 \mathrm{BC}-\mathrm{AD}$ \\
\end{tabular}

932 Analytic, the Research Laboratory for Archaeology and the History of Art (Oxford University), and by the Radiocarbon Laboratory, Institute of Physics - Centre for Science and Education, Silesian University of Technology. Calibration: OxCal 4.3.2, Intcal13, Sigma 2 (95.4\%).

Table 1b. Summary of the previous (2012) and revised (2019) OSL dating results on Jebel Moya sherds curated at the British Museum by the Research Laboratory for Archaeology and the History of Art (Oxford University).

\begin{tabular}{|l|l|l|l|l|}
\hline Provenience & \multicolumn{1}{|c|}{ Material } & Lab number & $\begin{array}{c}\text { Uncalibrated } \\
\text { bp }\end{array}$ & Calibrated BC \\
\hline $\begin{array}{c}\text { Jebel Tomat } \\
\text { Midden } \\
\text { periphery }\end{array}$ & Dark clay loam & SUA-67 & $4540+/-200$ & $3712-2679$ \\
\hline $\begin{array}{c}\text { Rabak } \\
\text { Level 2 }\end{array}$ & Shell & T-5132 & $4490+/-100$ & $3378-2909$ \\
\hline Level 6 & Shell & T-5133 & $6050+/-100$ & $5219-4722$ \\
\hline Level 15 & Shell & T-5134 & $6020+/-130$ & $5308-4686$ \\
\hline & & & & \\
\hline & & & & \\
\hline
\end{tabular}

Table X. Radiometric dates from Neolithic sites in the central and southern Gezira. Adapted from Clark and Stemler (1975: Table 1), and el Mahi and Haaland (1984: Table 1) calibrated using OxCal 4.3 (IntCal13, Sigma 2 (95.4\%) confidence interval). 
Author statement
Michael Brass is the first author

Author statement
Michael Brass is the first author

.

Author statement
Michael Brass is the first author

(

(

(2.

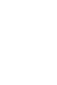

